\title{
Acupuncture-Point Stimulation for Postoperative Pain Control: A Systematic Review and Meta-Analysis of Randomized Controlled Trials
}

\author{
Xian-Liang Liu, ${ }^{1,2,3}$ Jing-Yu Tan, ${ }^{4}$ Alex Molassiotis, ${ }^{4}$ Lorna K. P. Suen, ${ }^{4}$ and Yan Shi ${ }^{1}$ \\ ${ }^{1} 10$ th People's Hospital of Tongji University, 301 Yanchang Road, Shanghai 200072, China \\ ${ }^{2}$ School of Nursing, Midwifery and Paramedicine, Australian Catholic University, 1100 Nudgee Road, Banyo, \\ Brisbane, QLD 4014, Australia \\ ${ }^{3}$ School of Nursing, Jinggangshan University, 28 Xueyuan Road, Jian 343009, China \\ ${ }^{4}$ School of Nursing, The Hong Kong Polytechnic University, Hung Hom, Kowloon, Hong Kong \\ Correspondence should be addressed to Yan Shi; nursingsytj@163.com
}

Received 3 April 2015; Revised 19 August 2015; Accepted 3 September 2015

Academic Editor: George David Baxter

Copyright (C) 2015 Xian-Liang Liu et al. This is an open access article distributed under the Creative Commons Attribution License, which permits unrestricted use, distribution, and reproduction in any medium, provided the original work is properly cited.

\begin{abstract}
The purpose of this study was to evaluate the effectiveness of Acupuncture-point stimulation (APS) in postoperative pain control compared with sham/placebo acupuncture or standard treatments (usual care or no treatment). Only randomized controlled trials (RCTs) were included. Meta-analysis results indicated that APS interventions improved VAS scores significantly and also reduced total morphine consumption. No serious APS-related adverse effects (AEs) were reported. There is Level I evidence for the effectiveness of body points plaster therapy and Level II evidence for body points electroacupuncture (EA), body points acupressure, body points APS for abdominal surgery patients, auricular points seed embedding, manual auricular acupuncture, and auricular EA. We obtained Level III evidence for body points APS in patients who underwent cardiac surgery and cesarean section and for auricular-point stimulation in patients who underwent abdominal surgery. There is insufficient evidence to conclude that APS is an effective postoperative pain therapy in surgical patients, although the evidence does support the conclusion that APS can reduce analgesic requirements without AEs. The best level of evidence was not adequate in most subgroups. Some limitations of this study may have affected the results, possibly leading to an overestimation of APS effects.
\end{abstract}

\section{Introduction}

Nearly $86 \%$ of surgery patients experience moderate to severe postoperative pain [1]. Depending on surgery type, as many as half of these patients go on to experience chronic postoperative pain [2]. Unsatisfactory pain control can limit patients' physical activities, prolong recovery time, and contribute to poor quality of life $[3,4]$. Pain may also increase postoperative complications, such as postoperative morbidity, and may extend the length of hospitalization, increasing health care costs $[5,6]$.

Administration of standard analgesics, which are considered generally safe and effective, remains the primary approach to postoperative pain management [7]. However, systemic analgesic administration can induce some adverse effects (AEs), such as nausea, vomiting, depressive symptoms, pruritus, urinary retention, gastrointestinal motility, and ileus $[8,9]$. AEs can impair physical and psychological wellbeing and, more seriously, may result in significant morbidity or even mortality [8-10]. To achieve better postoperative pain relief and reduce the requirement for analgesic medication, various nonpharmacological approaches, including educational intervention, relaxation, and acupuncture-point stimulation (APS), have been employed. APS has been lauded as a promising alternative method for achieving postoperative pain relief $[11,12]$.

APS is a widely used component of traditional Chinese medicine (TCM) together with full-body and auricular approaches [11]. In addition to the most popular methods of manual acupuncture and acupressure, APS can be achieved using modalities such as electrical acupuncture (EA) or laser stimulation and acupoint massage [12]. According to TCM 


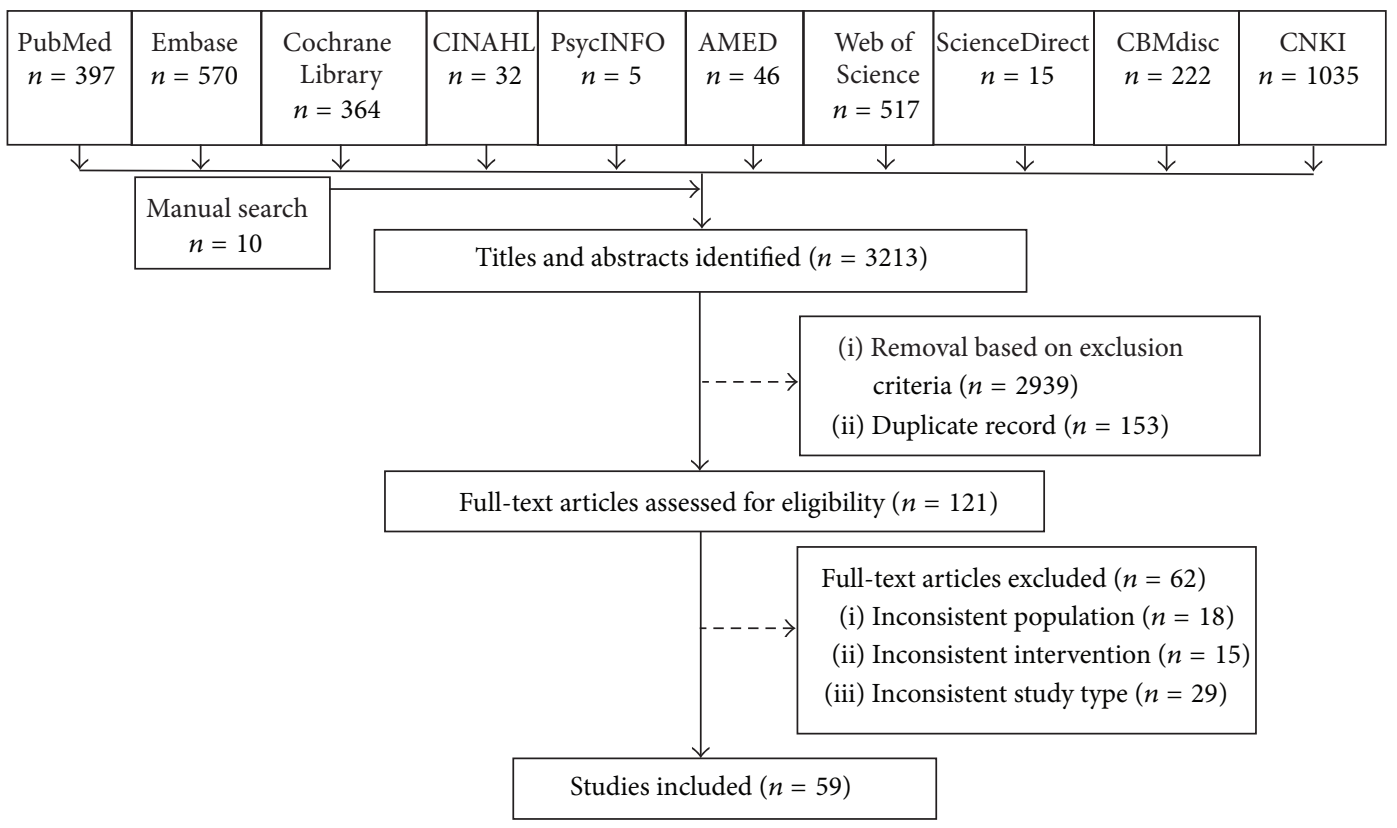

FIGURE 1: Flow chart of the study selection process. CINAHL, Cumulative Index to Nursing and Allied Health Literature; AMED, Allied and Complementary Medicine Database; CBMdisc, Chinese Biological Medical Literature Database; CNKI, China National Knowledge Infrastructure.

philosophy, the stimulation of target acupoints along meridians produces positive effects by rebalancing qi circulation in the body [13]. However, the existence of the qi meridian system, as described in TCM, has never been demonstrated scientifically [13]. Nevertheless, the management of various forms of pain remains a key purported benefit of APS [14, 15].

Many animal experiments and clinical studies have examined the therapeutic effects of APS [16]. Early studies showed that APS provided postoperative pain relief in comparison with control groups [17, 18]. Recently, several small trials $[3,19]$ demonstrated that APS can relieve pain and reduce analgesic requirements associated with hysterectomy and inguinal surgery. However, Sakurai et al. [20] failed to identify any significant change in pain intensity or morphine requirement in surgical patients undergoing acupressure. A prior systematic review found that acupuncture and related techniques aided postoperative pain control, but the quality of evidence was low due to the quality and quantity of included trials, and no subgroup analysis according to acupuncture type was performed [21]. The results of another systematic review conducted by Usichenko et al. [22] suggested that auricular acupuncture was a promising method of postoperative pain reduction, but the heterogeneity of primary studies precluded data synthesis and the evidence was insufficient to draw a definitive conclusion about the treatment's effectiveness. Following the 2008 publication of these reviews, several clinical trials were conducted to evaluate the efficacy of APS for postoperative pain management, generating new evidence on this topic [23-25].

The present systematic review and meta-analysis was conducted to evaluate the effectiveness of APS for pain control following surgical procedures. Therefore, in this study, current evidence generated by randomized controlled trials (RCTs) on the use of APS interventions for postoperative pain management was reviewed and analyzed. Data from patients receiving APS were compared with those from control groups receiving sham/placebo acupuncture, usual care, or no treatment. Compared with the previous literature, this systematic review and meta-analysis incorporates new evidence not previously synthesized and distinguishes between multiple types of APS for postoperative pain control.

\section{Methods}

2.1. Study Selection. As summarized in Figure 1, we performed a literature search to identify RCTs examining APS interventions in surgical patients with postoperative pain. We searched PubMed, Embase, PsycINFO, Allied and Complementary Medicine, Thomson Reuters Web of Science, ScienceDirect, China National Knowledge Infrastructure, Chinese Biological Medical Literature databases, the Cochrane Central Register of Controlled Trials, and the Cumulative Index to Nursing and Allied Health Literature from inception through 31 May 2014 (search strategies are described in Appendix). Additional relevant articles were identified by screening the reference lists of eligible studies and previous systematic reviews and by performing a manual search for articles published in the last 3 years in eight core TCM journals: Journal of Beijing University of Traditional Chinese Medicine, Journal of Nanjing University of Traditional Chinese Medicine (Natural Sciences), Chinese Journal of Traditional Chinese Medicine and Pharmacy, Chinese Journal of Information on Traditional Chinese Medicine, Chinese Journal of Basic Medicine in Traditional Chinese Medicine, Chinese Journal of 
Integrated Traditional and Western Medicine, Journal of Traditional Chinese Medicine, and Journal of Integrative Medicine. We also used PubMed's "related articles" function to identify additional potentially relevant studies. The electronic search had no language restriction. In the case that there were multiple publications from the same RCT, overlapping results were extracted from one publication.

Two reviewers assessed all potentially relevant articles independently. Disagreements regarding study selection were resolved by discussion, with strict adherence to the inclusion criteria. Studies were selected for inclusion based on the following criteria: (1) RCT; (2) adult (age $\geq 18$ years) participants with pain following any surgical procedure; (3) APS intervention (including full-body or auricular manual acupuncture or EA, acupressure, seed embedding, and plaster therapy) conducted by an acupuncturist, TCM practitioner, or other health care providers with qualification and/or training in acupuncture therapy; (4) control group receiving standard treatment (e.g., active pain control approach normally provided to surgical patients, including analgesia medication, nursing guidance, and other usual cares), sham/placebo APS (faked APS intervention), or no treatment (provision of usual postoperative care not involving active analgesic interventions); (5) primary outcome of pain intensity, measured by a valid self-reported instrument such as a visual analog scale (VAS), numerical rating scale (NRS), or verbal reporting; and (6) secondary outcomes of analgesic consumption and APS-related AEs (i.e., any adverse events resulting from APS intervention, minor (e.g., needling site pain), intermediate (e.g., bleeding and hematoma), or serious (pneumothorax and cardiac tamponade)).

In the study selection process, acupuncture was defined as the stimulation of specific acupuncture points along the skin of the body by using thin needles, with or without the application of heat, pressure, or laser light to these same points [12]. EA is similar to acupuncture but involves the use of devices (e.g., a wristwatch-like device and surface electrodes attached to a transcutaneous electrical nerve stimulation device) on acupoint [14]. Seed embedding was defined as an auricular acupressure process involving the embedding of magnetic beads or other seeds within skin-colored adhesive tape, which is placed on the auricular acupoints and retained in situ for several days [6]. In this systematic review, plaster therapy mainly referred to the use of capsicum plaster as an alternative to acupuncture [23].

2.2. Quality Assessment. Two reviewers conducted independent assessments of the methodological quality and risk of bias of each RCT using Cochrane Collaboration's risk of bias tool [26]. This tool provides for the assessment of seven domains: sequence generation, allocation concealment, blinding of participants and personnel, blinding of outcome assessment, incomplete outcome data, selective outcome reporting, and "other issues." Items were scored as showing low, high, or unclear risk of bias [26]. All disagreements on scoring were resolved by discussion. When a sufficient number of studies were available and a meaningful assessment of publication bias could be carried out, a funnel plot was constructed.
Adequate allocation concealment and blinding of outcome assessors were designated as key domains for this assessment, where key domains are not only more likely to influence bias magnitude and direction but also more likely to impact study results. Domain-based evaluation was employed as described in the Cochrane Handbook for Systematic Reviews of Interventions 5.0.2 (updated September 2009). The overall risk of bias was categorized as follows. An overall low risk of bias (plausible bias unlikely to alter the results) was identified when all key domains were assessed as having a low risk of bias. An overall unclear risk of bias (plausible bias that raises some doubt about the results) was identified when one or more key domains were assessed as having an unclear risk of bias. An overall high risk of bias (plausible bias that seriously weakens confidence in the results) was identified when one or more key domains were assessed as having a high risk of bias. Small studies have been shown to overestimate treatment effects and to be at increased risk of bias, allowing critical criteria such as blinding to be compromised. Studies were considered to be at low risk of bias if they had at least 200 participants, at unknown risk if they had 50 to 200 participants, and at high risk if they had fewer than 50 participants.

2.3. Data Extraction and Management. Two reviewers independently extracted the following data from included studies using a predefined form: authors, study design, setting, population and participant demographics, intervention characteristics (e.g., acupuncture type, acupoints used, and treatment duration), comparators, outcome measures and instruments, follow-up, and some numeric data (mainly the results of pain intensity and analgesic consumption). We contacted RCT authors by email to obtain data necessary for effect size estimation when such data were missing from publications (e.g., due to aggregated data reporting). When authors did not reply, outcome data presented only in figures and/or graphs were extracted when possible; these data were included in the analysis only when the two reviewers independently obtained the same results.

When a study reported multiple group comparisons (e.g., high electrical stimulation versus low electrical stimulation or usual care and preoperative acupuncture versus postoperative acupuncture or usual care), only data from the treatment group that received the more intensive and comprehensive postoperative intervention were included in the analysis. These data were compared with those from the control group.

2.4. Subgroup Analysis. When data were sufficient, subgroup analyses of different types of APS, surgery, and control groups were conducted. Analyses of APS type compared the use of acupoints on the body (EA, manual acupuncture, acupressure, and plaster therapy) and/or auricular points (EA, manual acupuncture, and seed embedding). A subgroup analysis of EA studies compared the use of different devices (e.g., a wristwatch-like device and surface electrodes attached to a transcutaneous electrical nerve stimulation device). Analyses of control group types compared APS with standard treatment (usual care and no treatment) or placebo/sham therapies. On the basis of whether body acupoints, auricular 
TABLE 1: Qualitative modified approach to grading of evidence.

\begin{tabular}{ll}
\hline Level & Description \\
\hline I & Evidence obtained from multiple relevant high quality randomized controlled trials \\
\hline II & $\begin{array}{l}\text { Evidence obtained from at least one relevant high quality randomized controlled trial or multiple relevant moderate } \\
\text { or low quality randomized controlled trials }\end{array}$ \\
\hline III & $\begin{array}{l}\text { Evidence obtained from at least one relevant moderate or low quality randomized controlled trial with multiple } \\
\text { relevant observational studies, or } \\
\text { evidence obtained from at least one relevant high quality nonrandomized trial or observational study with multiple } \\
\text { moderate or low quality observational studies }\end{array}$ \\
\hline IV & Evidence obtained from multiple moderate or low quality relevant observational studies \\
\hline V & Opinion or consensus of large group of clinicians and/or scientists \\
\hline
\end{tabular}

points, or integrative acupoints were stimulated, we also undertook subgroup analyses of surgery types, including abdominal, knee, oral, and cardiac surgeries.

2.5. Statistical Analysis. Meta-analysis was performed using Review Manager software (ver. 5.1; available from http://www .cochrane.org/). For continuous outcomes, mean differences with 95\% confidence intervals (CIs) were calculated as appropriate. When the same continuous outcome was assessed using different instruments, the standardized mean difference was calculated. For dichotomous outcomes, effect size variables, such as the relative risk (RR), were calculated. In the presence of significant heterogeneity $\left(\chi^{2}\right.$ test, $\left.P<0.1\right)$, random-effects model was used. Otherwise, fixed-effects model was applied. Descriptive analysis was used when data could not be converted or pooled.

Potential sources of heterogeneity in the outcomes examined are differences in the tool used to measure pain, population differences (e.g., surgery type, age, and sex), and differences in the comparator used (e.g., sham/placebo acupuncture, usual care, and no treatment). We assessed heterogeneity using the $I^{2}$ statistic, which describes the percentage of total variation across trials (low, 0-40\%; moderate, 3060\%; substantial, 50-90\%; considerable, 75-100\%; Chapter 9: Analysing Data and Undertaking Meta-Analyses; The Cochrane Collaboration 2011, available from http://www .cochrane-handbook.org/). To assess which RCTs affected the overall results, sensitivity analyses were performed for the entire sample and subgroups with significant heterogeneity. If heterogeneity was considerable, even with the random-effects model, best evidence synthesis was also used. The evidence was synthesized based on each subgroup. We employed a qualitative modified approach to grading of evidence, as summarized in Table 1.

\section{Results}

3.1. Characteristics of Included Trials. The database search yielded 3,203 publications. Manual searching of the reference lists and journals resulted in the retrieval of 10 additional citations. A total of 121 full-text articles were reviewed. After application of the inclusion criteria, 59 RCTs [3, 6, 14, 19, 20, 23-25, 28-78] conducted between 1986 and 2014 were included in the review (Figure 1; Tables 2-4). Nine publications were in Chinese, and the remaining 50 publications were in English. The included studies were conducted in mainland China, Hong Kong, Taiwan, the United States, Germany, Austria, South Korea, Japan, Iran, the United Kingdom, Brazil, Sweden, Singapore, Italy, and Turkey. Sixteen of these RCTs had three arms and three trials had four arms.

RCTs included in the analysis involved a total of 4,578 randomized patients, 4,402 of whom completed the respective studies (APS groups, $n=2,097$; control groups, $n=$ 2,$305 ; 96.16 \%$ completion rate). The average sample size was 73 (range, 18-150). Standardized anesthetic and postoperative analgesia regimens were used in all studies. Follow-up duration ranged widely from 7 days $[60,61]$ to 4 months [41]. Twenty-one RCTs stated that an intention-to-treat analysis was used.

Five types of APS were used: low- and/or high-intensity EA, manual needle acupuncture, seed embedding, acupressure, and plaster therapy. Preoperative and postoperative APS were used in two RCTs, and sham/placebo control was used in 36 studies. Chinese herbs were used as a control in a single study.

Acupuncture points on the body and/or auricular points were stimulated. Commonly used body points included Hegu (LI4), Sanyinjiao (SP6), Zusanli (ST36), and Nei guan (P6); commonly used auricular points included Shen Men (TF4), Stomach (CO4), and Lung (CO14).

3.2. Methodological Quality and Risk of Bias of Included Trials. The methodological quality of included studies is characterized in Figures 2 and 3. Eighteen (30.5\%) publications specifically stated that the outcome assessor was blinded and 23 (38.98\%) studies used adequate allocation concealment together with full methodological description. Twelve $(20.33 \%)$ studies were rated highly in both of these domains and were deemed to be at low risk of overall bias. Forty-two studies (71.19\%) were deemed to be at unclear risk of overall bias. Finally, five studies (8.5\%) were deemed to be at high overall risk of bias. Allocation concealment was not reported or was described poorly in $24(40.67 \%)$ studies. According to the number of participants, no studies were considered to be at low risk of bias ( $\geq 200$ participants), 42 studies $(71.19 \%)$ were at unknown risk of bias (50-200 participants), and 17 studies (28.81\%) were at high risk of bias 


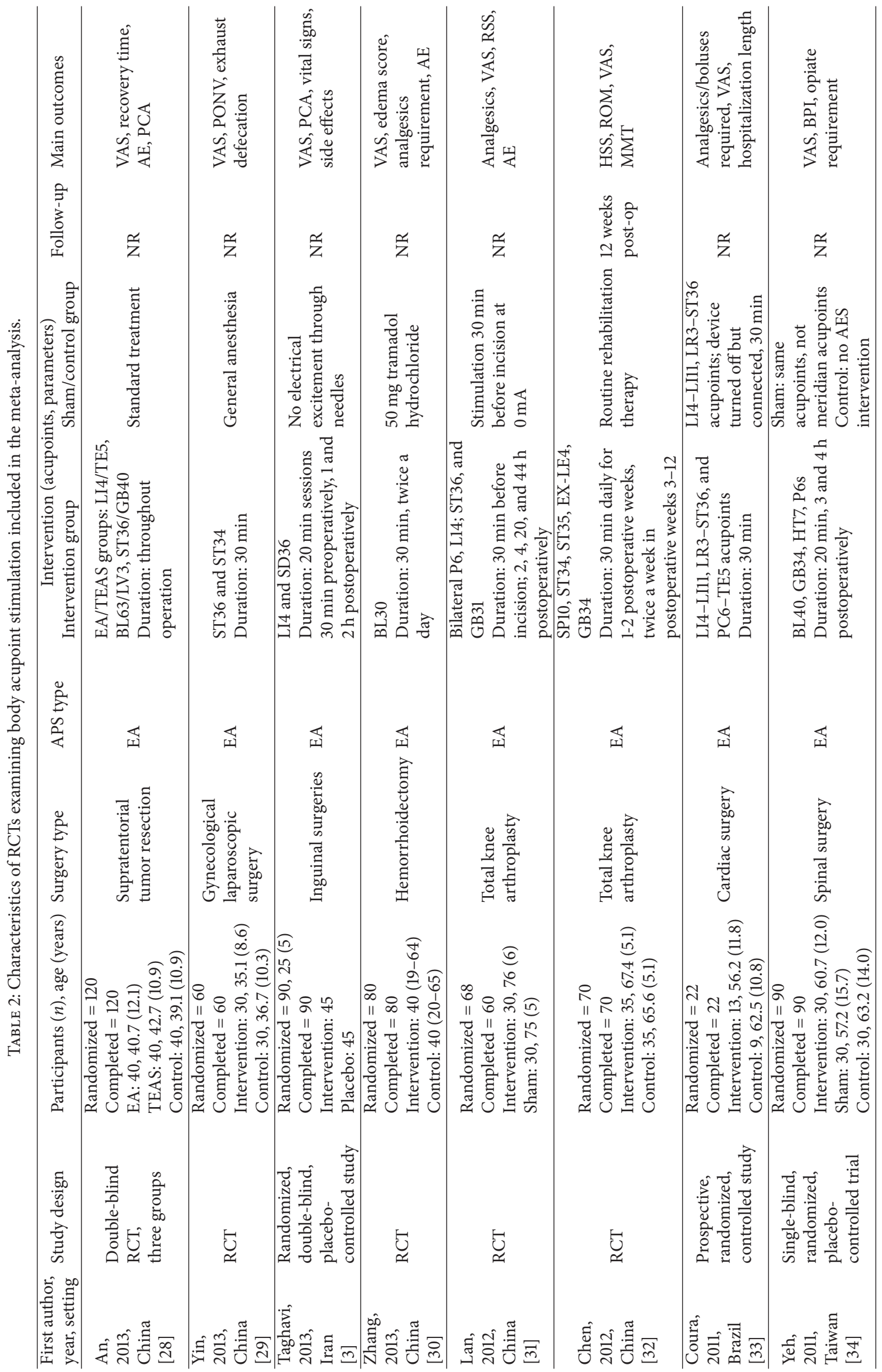




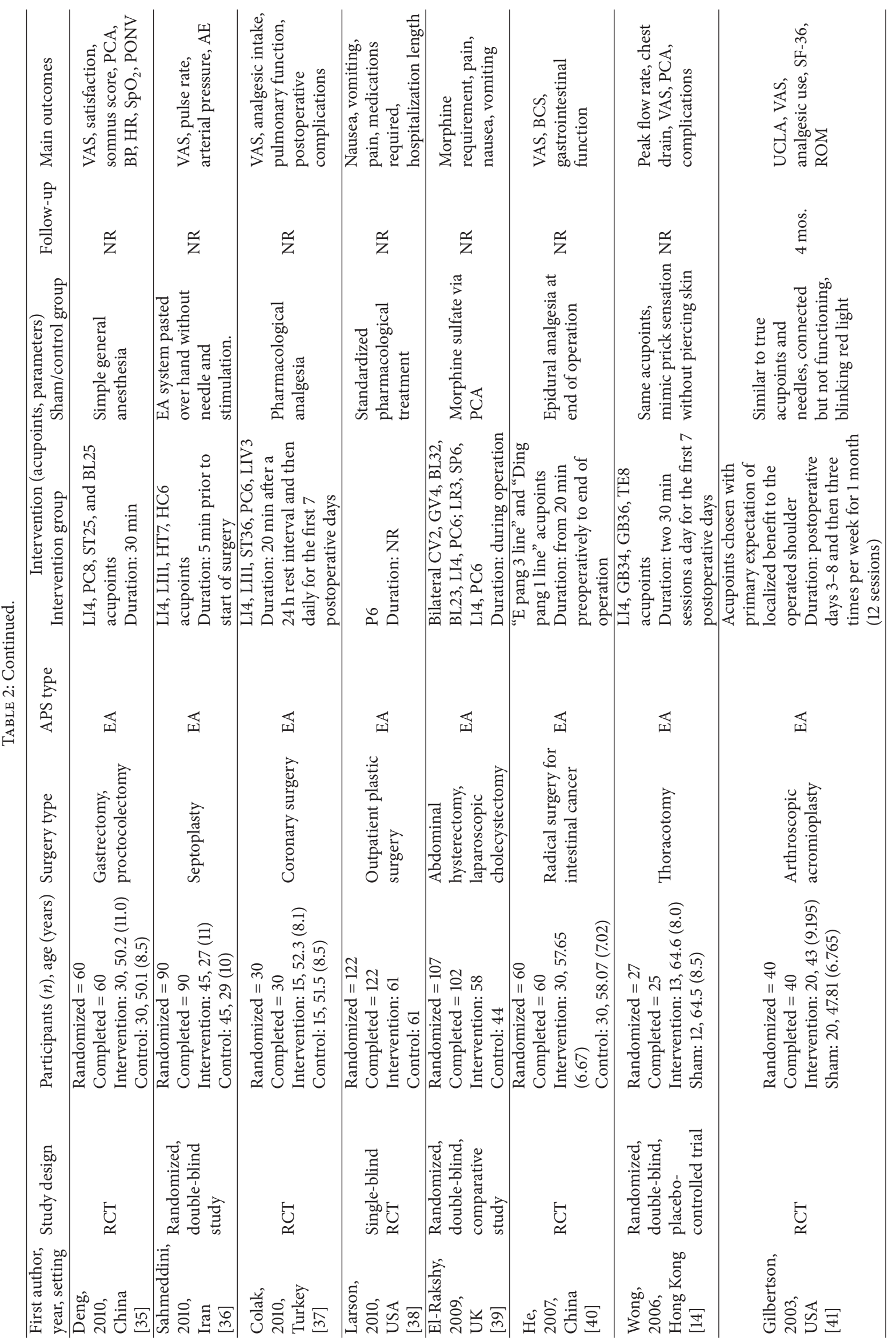




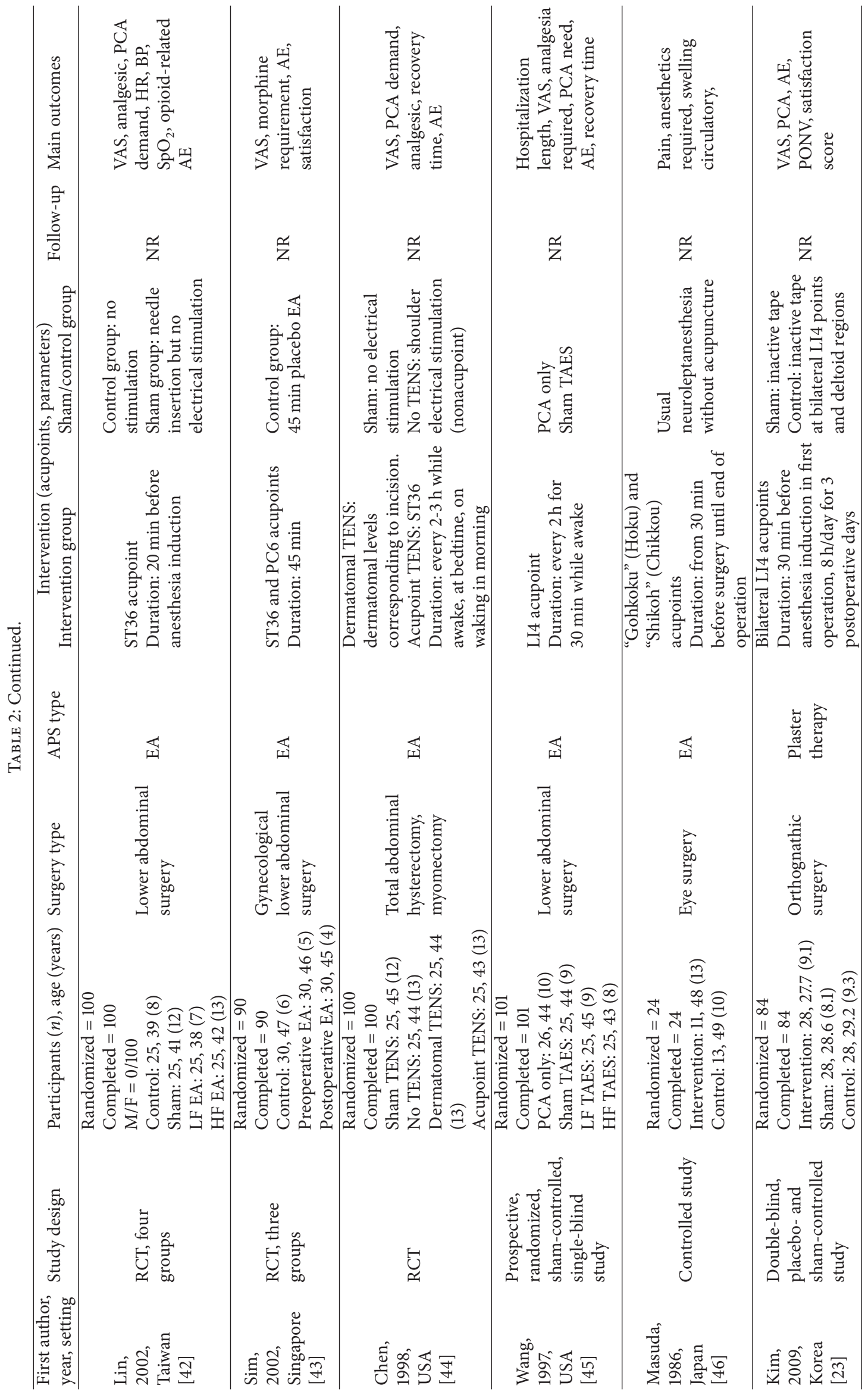




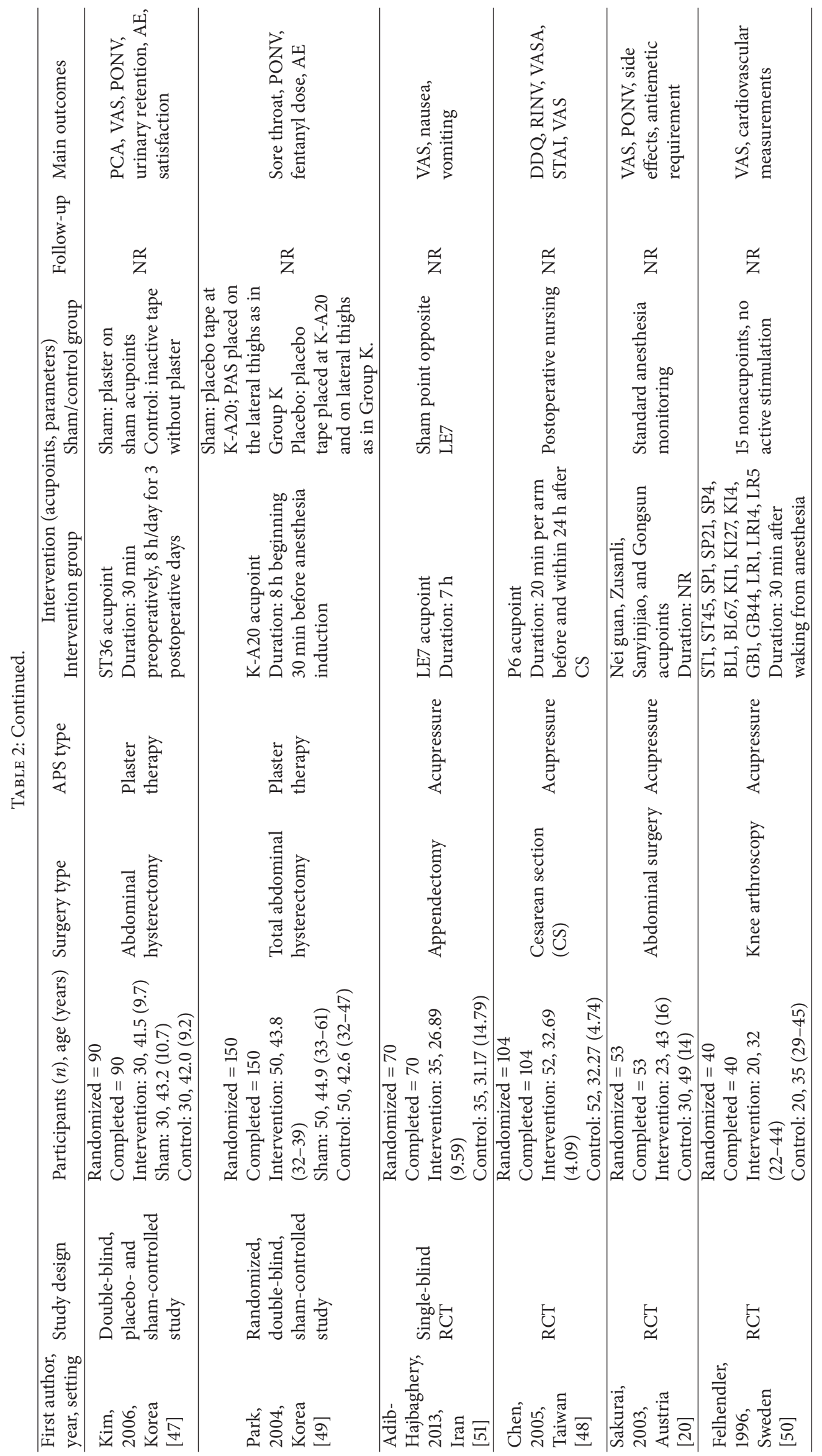




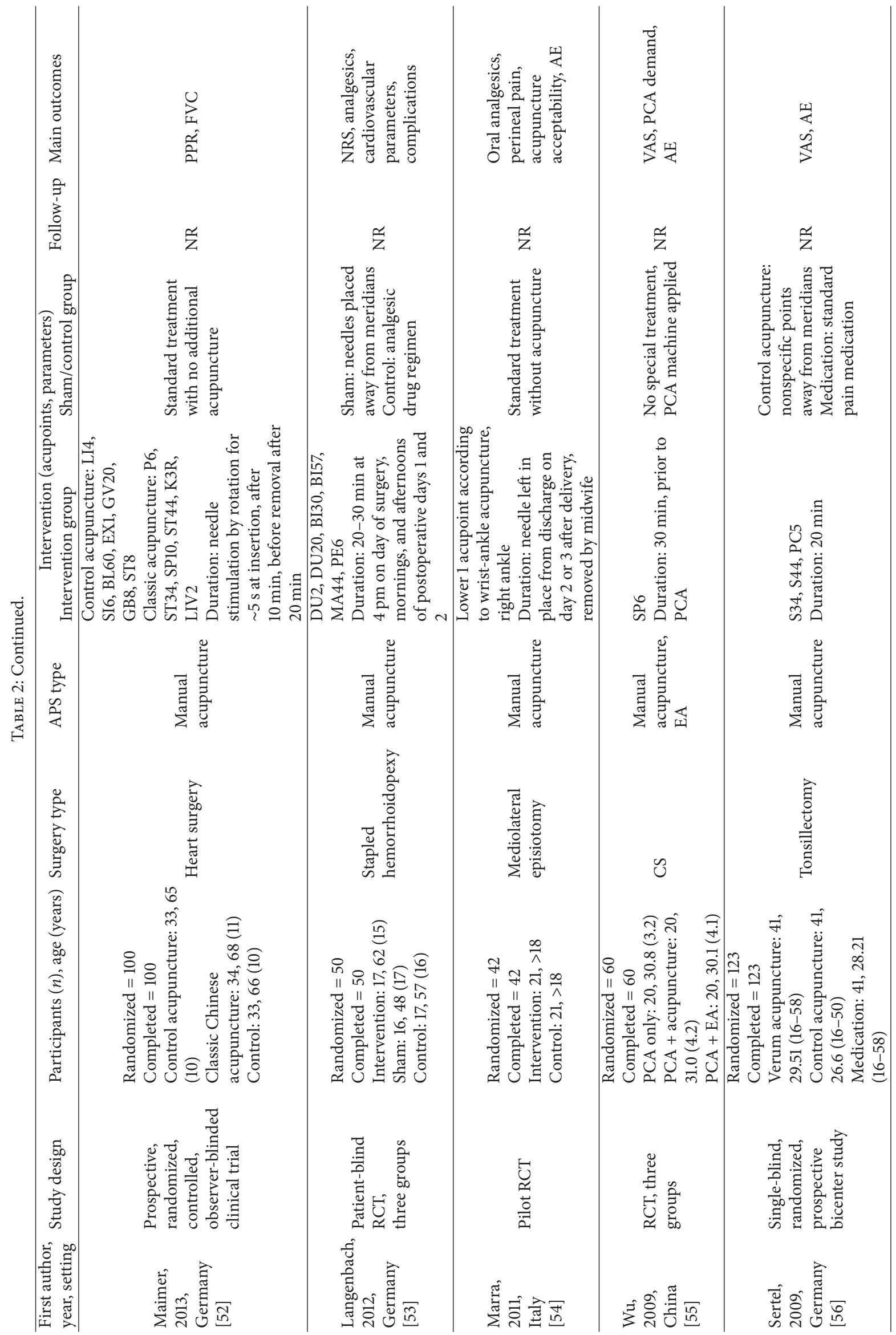




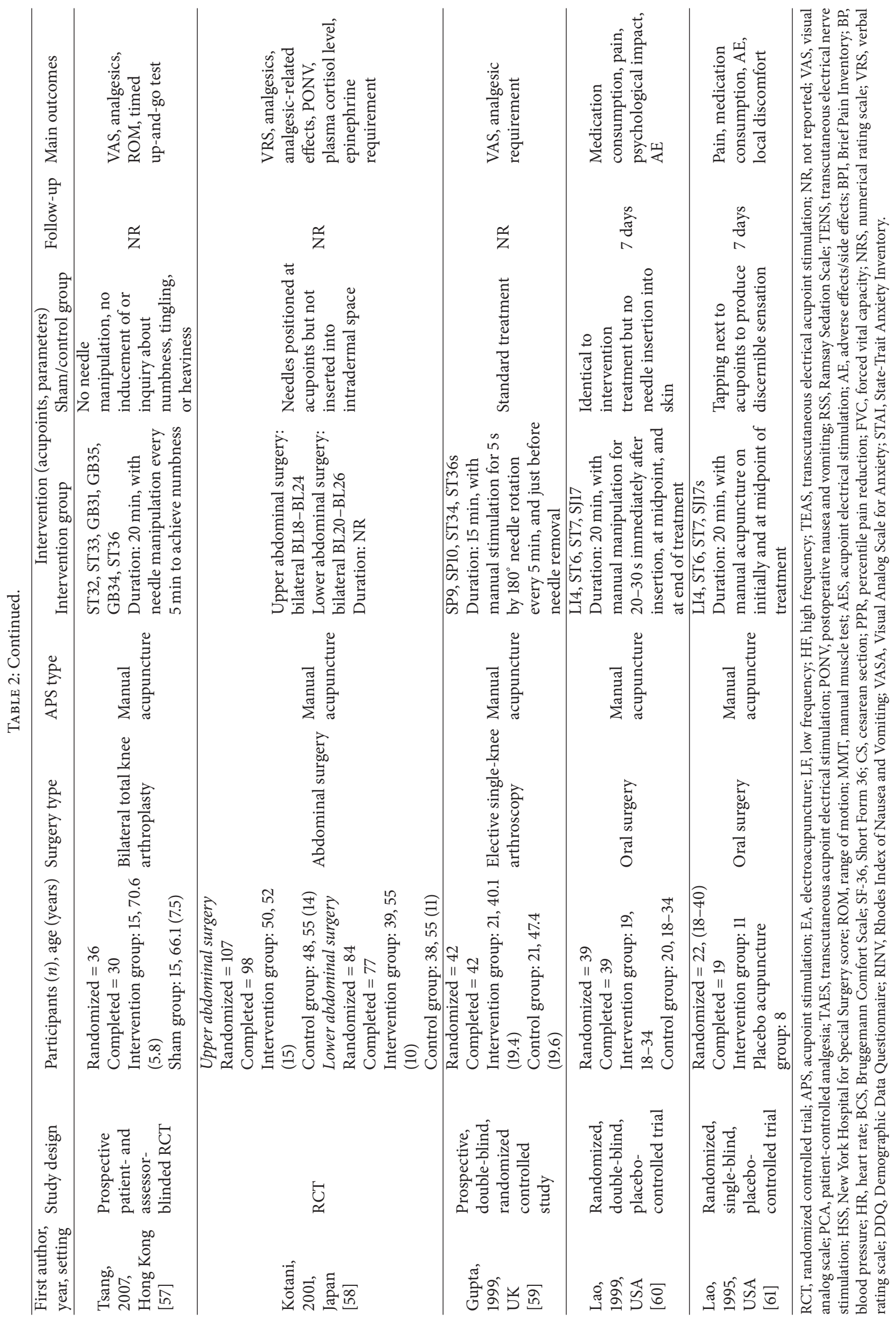




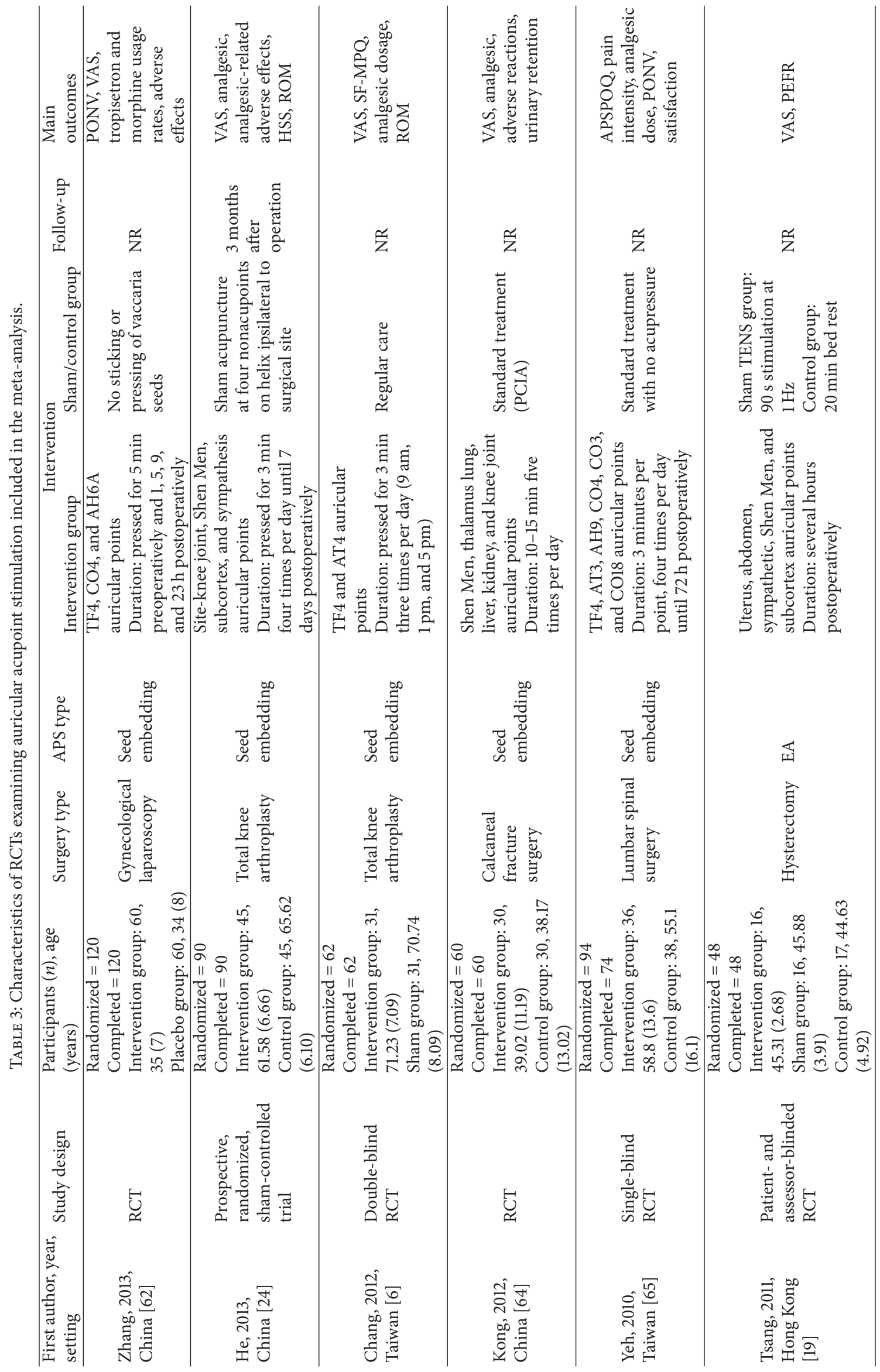




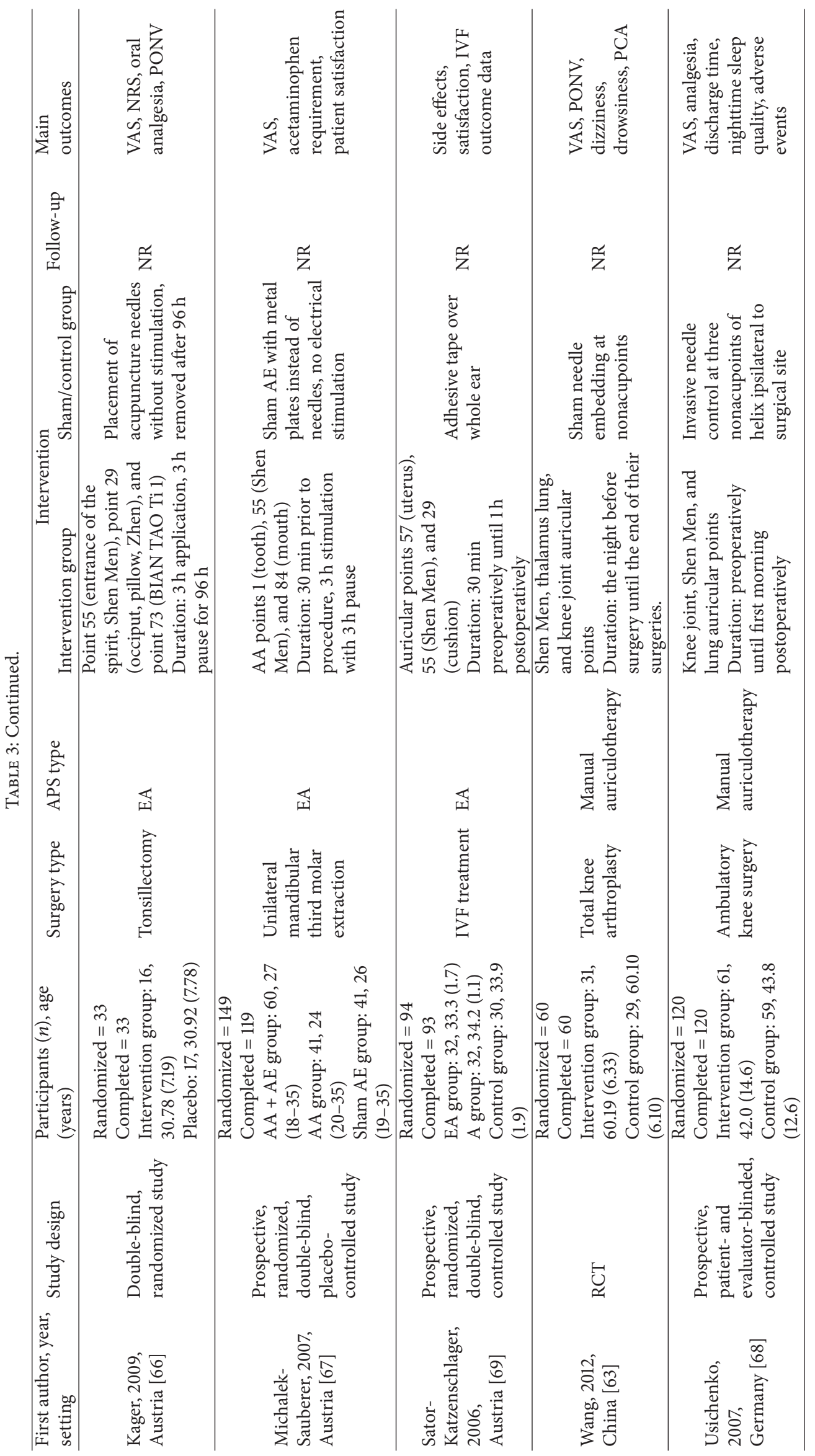




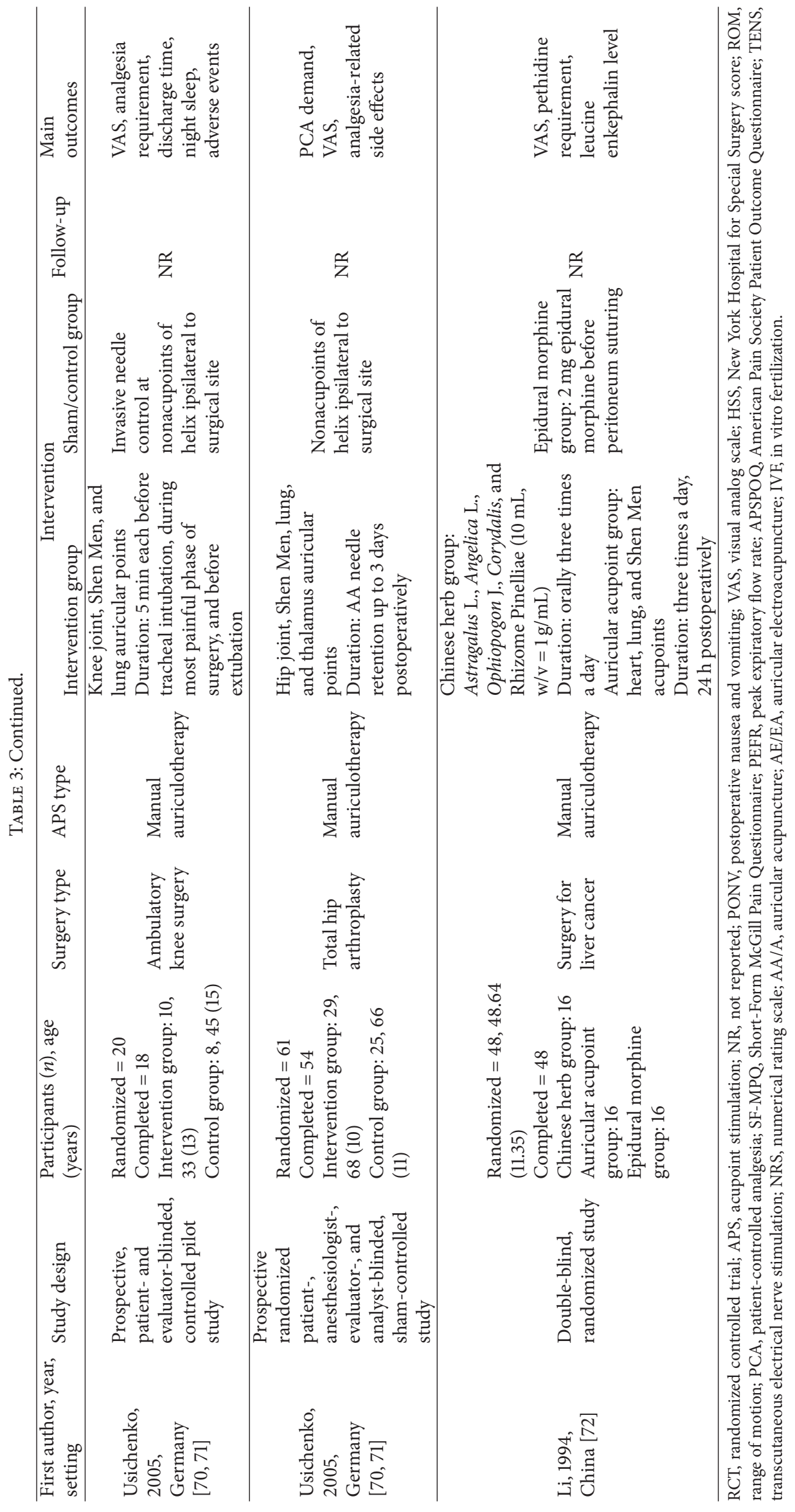




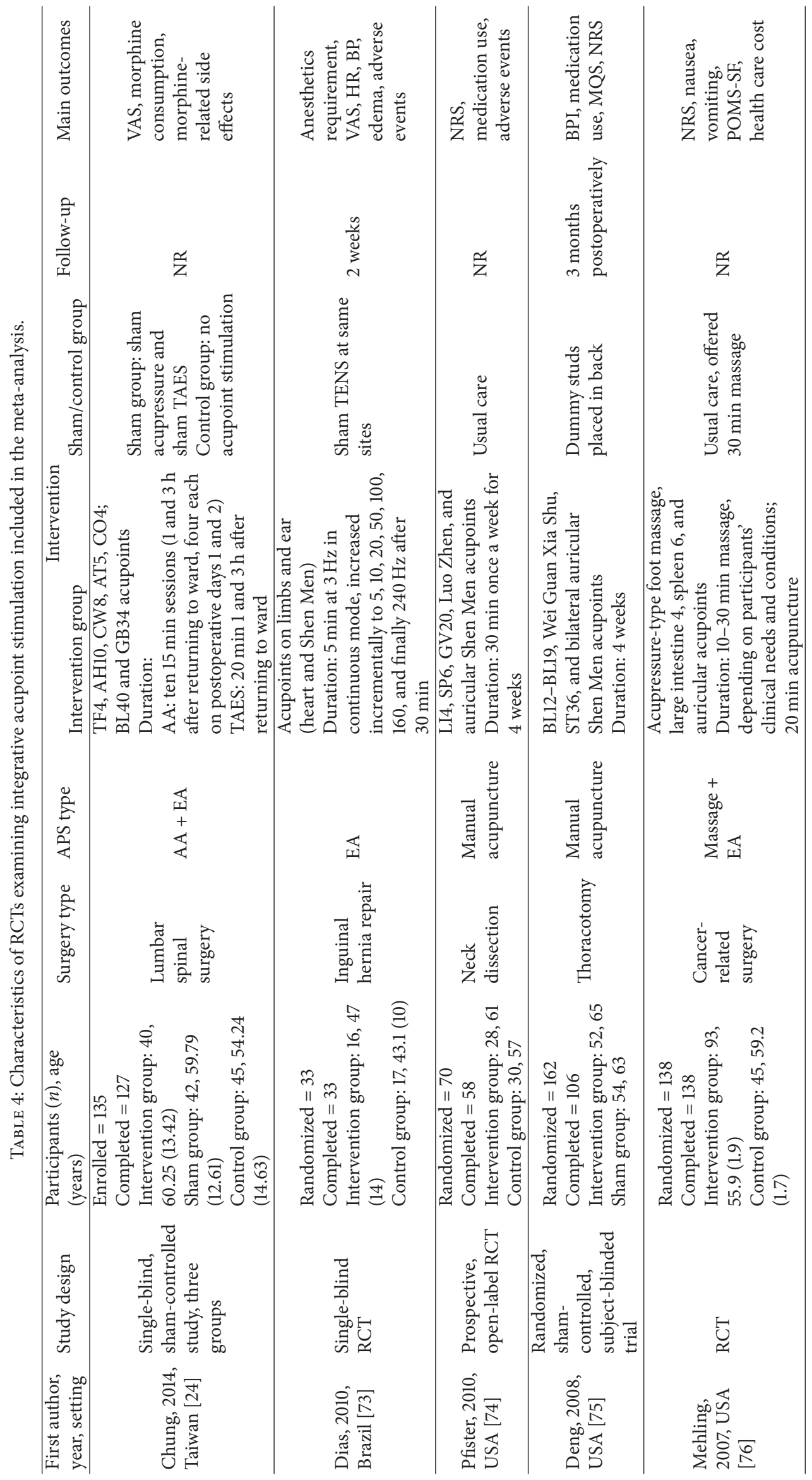




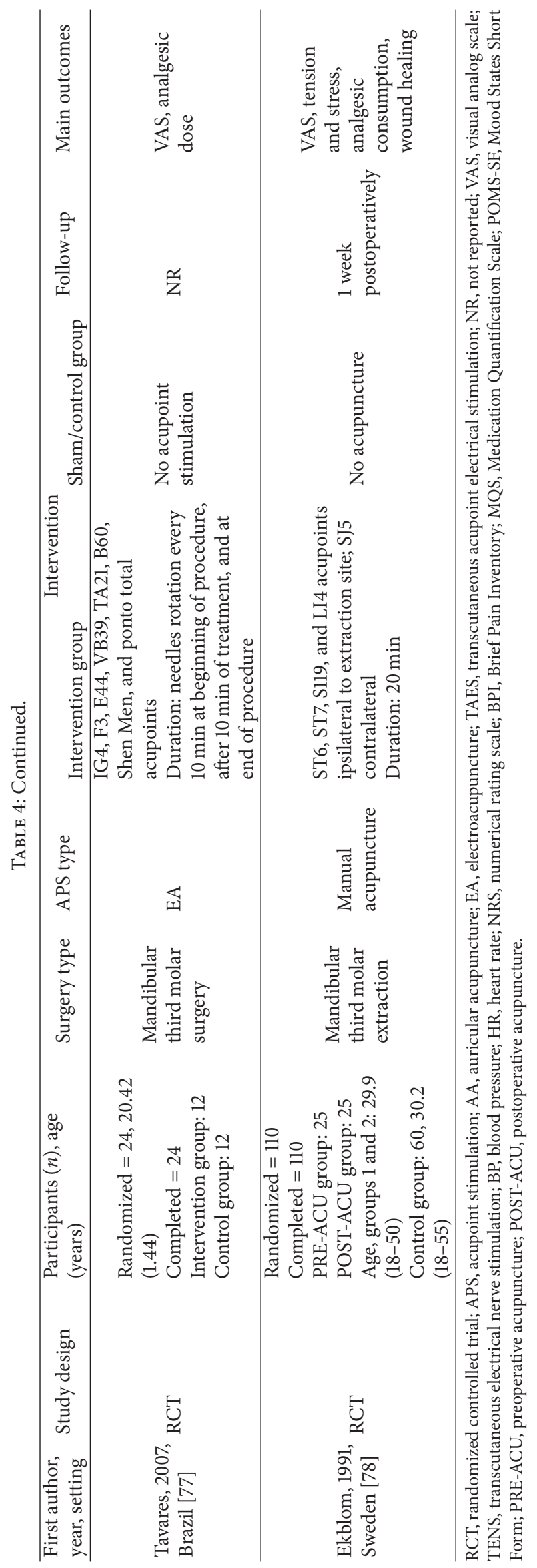




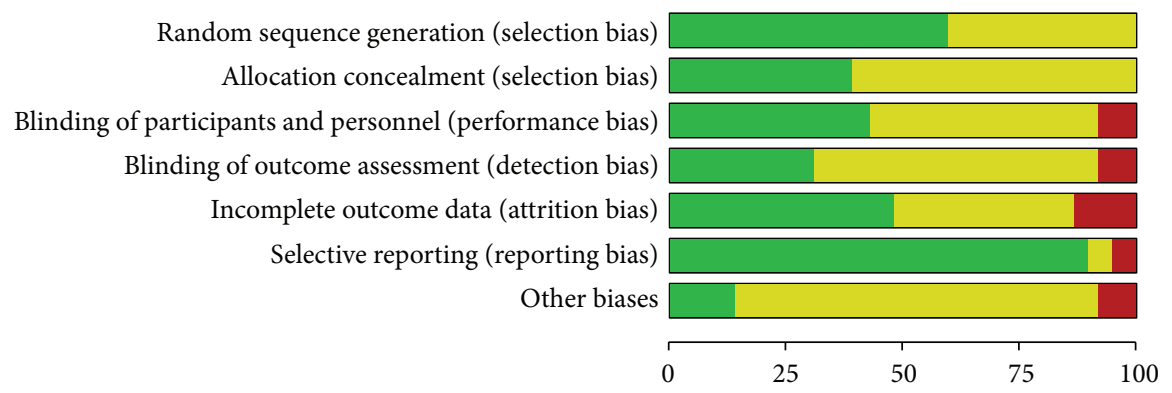

(\%)

Low risk of bias

Unclear risk of bias

High risk of bias

FIGURE 2: Methodological quality of included studies. Each methodological quality item was qualitatively assessed and is presented as a percentage across all included studies.

( $<50$ participants). Visual inspection of funnel plots revealed some substantial asymmetry in comparisons (Figure 4).

\subsection{Meta-Analysis and Descriptive Analysis of Outcomes}

3.3.1. Postoperative Pain. The results of the meta-analysis are reported in Table 5. The RCTs evaluated the efficacy of APS for postoperative pain relief by using VAS scores $(n=45)$, NRS scores $(n=5)[53,66,74-76]$, Brief Pain Inventory (BPI) scores $(n=2)[34,75]$, and a four-point scale and pain-free time to evaluate postoperative pain $(n=3)[58,60,61]$. Two of the five RCTs $[53,74]$ that used NRS scores reported a significant difference between groups, but these data could not be included in the meta-analysis due to clinical and statistical heterogeneity. One of two RCTs $[34,75]$ that used BPI scores reported a significant difference between the intervention and control groups, but these data could not be included in the meta-analysis due to different data modes. Three trials [58, $60,61]$ using a four-point scale reported that the intervention reduced pain intensity, but one of these studies [58] reported that total or partial pain relief did not differ significantly between the groups. Two trials $[60,61]$ reported that the APS intervention increased the duration of postoperative painfree status compared with that of the control groups.

Thirty-eight RCTs used body points for stimulation. Subgroup analyses according to control treatment and meta-analysis of 20 RCTs indicated that APS interventions improved VAS scores significantly in comparison with standard treatment and sham/placebo control (both $P<0.00001$; Table 5). Similarly, pooled results from 24 trials showed that body APS significantly improved VAS scores in comparison with all control groups $(P<0.00001)$, and subgroup analyses revealed similar improvement compared with standard treatment $(P<0.00001)$ and sham/placebo control $(P<0.0001$; Table 5). The evidence for body points APS reducing postoperative pain intensity in surgery patients was determined to be of Level I quality based on six overall high quality RCTs $[14,23,47,49,52,58]$. A meta-analysis of pooled results and subgroup analyses of body EA, as well as invasive and noninvasive forms of this treatment, yielded similar results (Table 5). The evidence for body points EA reducing postoperative pain intensity in surgery patients was determined to be of Level II quality based on one overall high quality RCT [14]. High-frequency EA was found to be more effective than low-frequency EA $[42,45]$. Pooled results from three RCTs examining acupressure $[48,50,51]$ showed a significant difference in VAS scores between intervention and control groups ( $P=0.01$; Table 5), although a fourth study [20] not included in the meta-analysis showed no such difference. The evidence for body points acupressure reducing postoperative pain intensity in surgery patients was determined to be of Level II quality based on three moderate quality RCTs $[20,48,50]$ and one low quality RCT [51]. Synthesis of data from two RCTs [23, 47] examining plaster therapy showed a significant reduction in pain intensity compared with standard treatment $(P<0.00001)$ and sham controls $(P<0.0001$; Table 5), and one other study [49] examining this treatment obtained similar results. The evidence for body points plaster therapy reducing postoperative pain intensity in surgery patients was determined to be of Level I quality based on three overall high quality RCTs [23, 47, 49].

In contrast, meta-analysis including three studies [55-57] revealed no significant effect of manual acupuncture on VAS score. Four $[55,57,59,60]$ of 10 RCTs examining manual acupuncture reported no difference in pain score between the intervention and control groups, whereas the remaining six studies found that this treatment reduced postoperative pain intensity $(P<0.05)$.

Twelve RCTs used body point stimulation for patients with abdominal surgery. The pooled results from eight trials $[29,35,40,42,43,45,47,51]$ showed that body APS significantly improved VAS scores in these patients $(P=0.0006)$. The evidence for body points APS reducing postoperative pain intensity in patients who had undergone abdominal surgery was determined to be of Level II quality based on one overall high quality RCT [47]. Five [31, 32, 50, 57, 59] RCTs used body point stimulation for patients with knee surgery. Pooled results from four trials [31, 32, 50, 57] showed that 


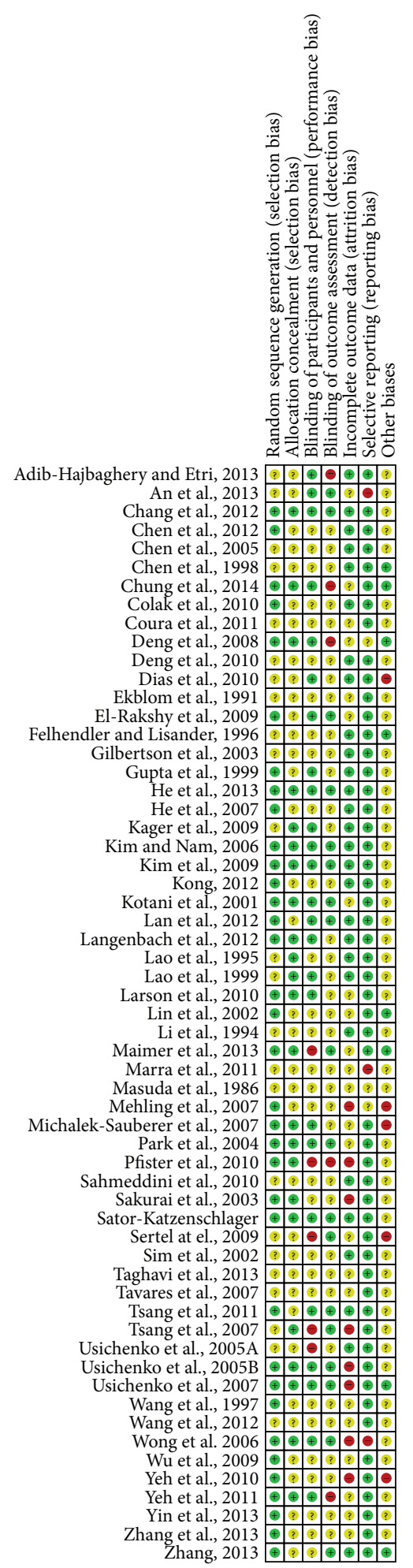

FIGURE 3: Risk of bias in the included studies. Each bias item was qualitatively assessed.

body APS did not significantly improve VAS scores for these patients $(P=0.16)$. Each of two RCTs used body point stimulation for patients with oral surgery $[60,61]$, cardiac surgery $[33,37]$, hemorrhoid operation $[30,53]$, or cesarean section $[48,55]$. Pooled results from two trials showed that body APS significantly improved VAS scores for patients undergoing cardiac surgery $[33,37](P=0.002)$ or cesarean section $[48,55](P<0.00001)$. The evidence for body points APS reducing pain intensity in patients who underwent cardiac surgery and cesarean section surgery was determined to be of 


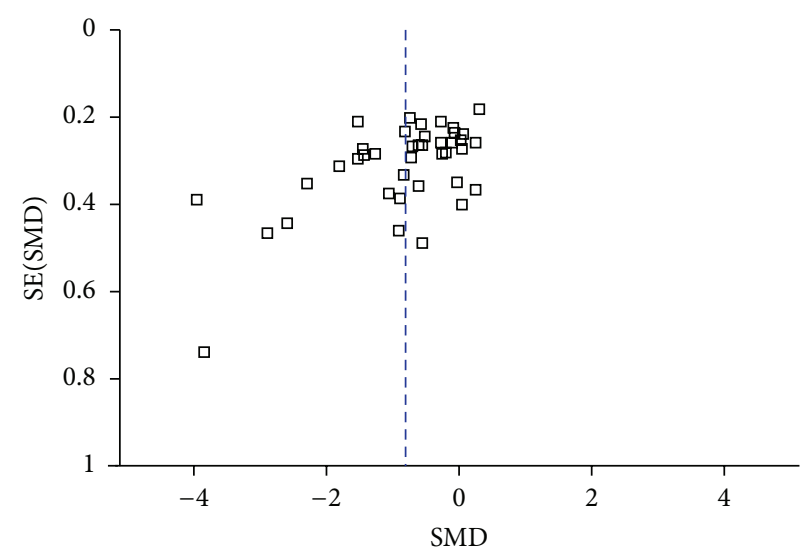

FIGURE 4: Funnel plot.

Level III quality based on two moderate quality RCTs $[33,37]$. Other studies could not be included in the meta-analyses due to insufficient data and the different types of surgery.

Fourteen [6, 19, 24, 62-72] RCTs used auricular points for stimulation. Data synthesis from 12 studies showed significantly lower VAS scores in intervention groups than in all types of control group $(P=0.001)$, and similar results were obtained in comparison with standard treatment $(P=$ $0.04)$ and sham/placebo control $(P=0.02)$ groups (Table 5$)$. The evidence for auricular points APS reducing postoperative pain intensity was determined to be of Level I quality based on six overall high quality RCTs $[6,24,68,69,71]$. Metaanalysis of data from five studies $[6,24,62,64,65]$ examining seed embedding also showed a significant effect on VAS score in comparison with all control groups $(P=0.02$; Table 5$)$. The evidence for auricular points seed embedding reducing postoperative pain intensity was determined to be of Level II quality based on two overall high quality RCTs $[6,24]$. Two studies $[6,65]$ of this auricular APS technique found a gradual reduction in pain, but no significant difference, according to VAS and Short-Form McGill Pain Questionnaire scores. One study [63] of manual auricular acupuncture data reported a significant difference in VAS score, and another study [72] showed a positive trend toward less pain in the intervention group, but meta-analysis of data from four studies $[63,68,70$, 71] showed that this auricular APS type was not associated with significant pain reduction. The evidence for manual auricular acupuncture reducing postoperative pain intensity in surgery patients was determined to be of Level II quality based on two overall high quality RCTs [68, 71]. Metaanalysis of auricular EA data from three studies [19, 66, 69] showed a significant reduction in VAS scores (including those reflecting pain at rest and on huffing and coughing; $P<0.0001$ ), although two of the four RCTs examining this treatment found no significant difference due to low pain intensity in intervention groups. The evidence for auricular EA reducing postoperative pain intensity in surgery patients was determined to be of Level II quality based on one overall high quality RCT [69].

Five $[6,24,63,68,70]$ RCTs used auricular point stimulation for patients with knee surgery. Pooled results from five trials $[6,24,63,68,70]$ showed that auricular point APS did not significantly improve VAS scores for these patients $(P=0.20)$. Two $[19,62]$ RCTs used auricular point stimulation for patients with abdominal surgery. Pooled results from both trials $[19,62]$ showed that auricular point APS significantly improved VAS scores for these patients $(P=0.01)$. The evidence for auricular point stimulation reducing postoperative pain intensity in abdominal surgery patients was determined to be of Level III quality based on two moderate quality RCTs $[19,62]$.

Seven RCTs [25, 73-78] used integrative APS (combined stimulation of body and auricular points) and evaluated postoperative pain relief using VAS $(n=4)$ and NRS $(n=3)$ scores. This meta-analysis showed a significant effect of integrative APS on pain intensity based on pooled VAS and NRS scores $(P=0.03$; Table 5) [73-78]. The evidence for integrative APS reducing postoperative pain in surgery patients was determined to be of Level II quality based on five moderate quality $[25,73,76-78]$ and two low quality $[74,75]$ RCTs. Two [77, 78] RCTs used integrative APS for patients with oral surgery. Pooled results from both trials [77, 78] showed that integrative APS did not significantly improve the VAS scores for these patients $(P=0.34)$.

3.3.2. Analgesic Requirement. Forty-three RCTs measured analgesic use, and most studies documented a lesser analgesic requirement in APS intervention groups than in control groups. Meta-analysis of data from six RCTs [3, 25, 34, 42, $43,55]$ showed a significant reduction in total morphine consumption in intervention groups compared to the control groups $(P=0.0001)$. Similar results were obtained in the comparison of intervention and sham/placebo control groups $(P<0.00001 ;$ Table 5). In addition, Lin et al. [42] reported that the morphine requirement after high-frequency EA was decreased by $31 \%$ compared with that after low-frequency EA. The evidence for APS reducing analgesic requirement in surgery patients was determined to be of Level I quality based on multiple overall high quality RCTs.

3.3.3. AEs. No serious AEs were associated with APS, and patients were reported to tolerate the intervention well in 
TABLE 6: Sensitivity analysis results.

\begin{tabular}{|c|c|c|c|c|c|}
\hline $\begin{array}{l}\text { Presensitivity } \\
\text { analysis } \\
\text { statistical method }\end{array}$ & Heterogeneity & Sensitivity analysis & $\begin{array}{l}\text { Number of } \\
\text { trials } \\
\text { (patient } N \text { ) }\end{array}$ & $\begin{array}{c}\text { Postsensitivity } \\
\text { analysis } \\
\text { statistical method }\end{array}$ & Heterogeneity \\
\hline \multicolumn{6}{|c|}{ Effects of interventions, all body modalities versus standard treatment control (VAS) } \\
\hline $\begin{array}{l}\text { Random, }-1.08 \\
(-1.54,-0.61) \\
\end{array}$ & $\begin{array}{c}P<0.00001 \\
\quad I^{2}=90 \%\end{array}$ & $\begin{array}{l}\text { Remove Kim et al. } 2009 \text { [23] } \\
\text { and Sertel et al. } 2009 \text { [56] }\end{array}$ & $12(755)$ & $\begin{array}{c}\text { Random, }-0.74 \\
(-1.05,-0.43)\end{array}$ & $\begin{array}{c}P<0.00001 \\
I^{2}=76 \%\end{array}$ \\
\hline \multicolumn{6}{|c|}{ Effects of invasive body electroacupuncture versus all controls (VAS) } \\
\hline $\begin{array}{l}\text { Random, }-0.67 \\
(-1.11,-0.24) \\
\end{array}$ & $\begin{array}{c}P<0.00001 \\
\quad I^{2}=83 \%\end{array}$ & $\begin{array}{l}\text { Remove Gilbertson et al. } 2003 \\
{[41]}\end{array}$ & $9(505)$ & $\begin{array}{c}\text { Random, }-0.47 \\
(-0.79,-0.15)\end{array}$ & $\begin{array}{l}P=0.002 \\
I^{2}=68 \%\end{array}$ \\
\hline \multicolumn{6}{|c|}{ Effects of body electroacupuncture versus standard treatment (VAS) } \\
\hline $\begin{array}{l}\text { Random, }-0.68 \\
(-1.04,-0.32) \\
\end{array}$ & $\begin{array}{l}P<0.00001 \\
\quad I^{2}=78 \%\end{array}$ & $\begin{array}{l}\text { Remove Deng et al. } 2010 \text { [35] } \\
\text { and Chen et al. } 2012 \text { [32] }\end{array}$ & $8(461)$ & $\begin{array}{l}\text { Fixed, }-0.38 \\
(-0.57,-0.20)\end{array}$ & $\begin{array}{l}P=0.33 \\
I^{2}=13 \%\end{array}$ \\
\hline \multicolumn{6}{|c|}{ Effects of body electroacupuncture versus sham/placebo control (VAS) } \\
\hline $\begin{array}{l}\text { Random, }-0.67 \\
(-1.13,-0.22) \\
\end{array}$ & $\begin{array}{c}P<0.00001 \\
I^{2}=80 \%\end{array}$ & $\begin{array}{c}\text { Remove Gilbertson et al. } 2003 \\
{[41]}\end{array}$ & $8(377)$ & $\begin{array}{l}\text { Fixed, }-0.42 \\
(-0.62,-0.21)\end{array}$ & $\begin{array}{l}P=0.08 \\
I^{2}=44 \%\end{array}$ \\
\hline \multicolumn{6}{|c|}{ Effects of body acupressure versus standard treatment (VAS) } \\
\hline $\begin{array}{l}\text { Random, }-1.44 \\
(-2.56,-0.33)\end{array}$ & $\begin{array}{c}P<0.00001 \\
I^{2}=92 \%\end{array}$ & $\begin{array}{l}\text { Remove Adib-Hajbaghery and } \\
\text { Etri } 2013 \text { [51] }\end{array}$ & $2(144)$ & $\begin{array}{l}\text { Fixed, }-1.93 \\
(-2.33,-1.53)\end{array}$ & $\begin{array}{l}P=0.75 \\
I^{2}=0 \%\end{array}$ \\
\hline \multicolumn{6}{|c|}{ Overall effects of auricular interventions, all modalities versus sham/placebo control (VAS) } \\
\hline $\begin{array}{l}\text { Random, }-0.56 \\
(-1.05,-0.07)\end{array}$ & $\begin{array}{c}P<0.00001 \\
I^{2}=87 \%\end{array}$ & $\begin{array}{l}\text { Remove Zhang } 2013[62] \text { and } \\
\text { Sator-Katzenschlager et al. } \\
2006 \text { [69] }\end{array}$ & $7(409)$ & $\begin{array}{l}\text { Random, }-0.23 \\
(-0.54,0.09)\end{array}$ & $\begin{array}{l}P=0.04 \\
I^{2}=55 \%\end{array}$ \\
\hline \multicolumn{6}{|c|}{ Effects of auricular manual acupuncture versus sham/placebo control (VAS) } \\
\hline $\begin{array}{l}\text { Random, }-0.16 \\
(-0.62,0.30) \\
\end{array}$ & $\begin{array}{l}P=0.04 \\
I^{2}=64 \%\end{array}$ & $\begin{array}{c}\text { Remove Usichenko et al. } 2007 \\
{[68]}\end{array}$ & $3(132)$ & $\begin{array}{l}\text { Fixed, }-0.34 \\
(-0.69,0.00)\end{array}$ & $\begin{array}{l}P=0.25 \\
I^{2}=27 \%\end{array}$ \\
\hline \multicolumn{6}{|c|}{ Overall effects of integrative interventions versus all controls (VAS and NRS) } \\
\hline $\begin{array}{l}\text { Random, }-0.61 \\
(-1.14,-0.07) \\
\end{array}$ & $\begin{array}{c}P<0.0001 \\
I^{2}=84 \%\end{array}$ & $\begin{array}{c}\text { Remove Tavares et al. } 2007 \\
\text { [77] }\end{array}$ & $5(420)$ & $\begin{array}{c}\text { Fixed, }-0.29 \\
(-0.49,-0.09)\end{array}$ & $\begin{array}{l}P=0.12 \\
I^{2}=46 \%\end{array}$ \\
\hline
\end{tabular}

the 21 RCTs that reported on this outcome. Reported minor AEs included temporary increased pain [74], localized pain or discomfort at insertion sites [60,71], minor bruising or bleeding [74], constitutional symptoms [74], and a mild burning sensation with erythema [23, 47, 49]. MichalekSauberer et al. [67] stated that $38 \%$ of patients reported minimal side effects of acupuncture, most commonly fatigue $(16 \%)$ and ear pain (10\%).

3.4. Sensitivity and Heterogeneity. Given the detection of obvious heterogeneity $\left(I^{2}>50 \%\right)$ in meta-analyses, we conducted a sensitivity analysis to remove studies with a greater risk of bias. The results are presented in Table $6 . I^{2}$ values were decreased substantially by the removal of such trials in most comparisons.

\section{Discussion}

In this review, it was determined that there is insufficient evidence thus far to conclude that APS is an effective nonpharmacological approach to the reduction of postoperative pain intensity for surgery patients, although the evidence did show a reduced analgesic requirement with no significant adverse effects in surgery patients. The results may have been affected by some limitations of this study, such as the wide variability of interventions and participants, absence of follow-up evaluation in most included trials, and the often mediocre methodological quality of the included studies. These factors contributed to the high heterogeneity of the data, which limits the strength of the evidence. No studies were considered to be at low risk of bias ( $\geq 200$ participants) based on the number of participants. These factors may have led to overestimations of APS efficacy.

Given the intensity of surgical trauma, postoperative pain is inevitable and it is deemed to be a serious problem. If this pain is not managed effectively, it can contribute to several clinical risks and affect patients' physical and psychological wellbeing; potential effects include emotional distress, infection, increased myocardial oxygen consumption, and prolonged hospitalization. Associated pathological changes can harm organs and lead to abnormal function [31, 33]. Reduction of postoperative pain is therefore essential.

Our meta-analysis of overall effects from 39 trials showed that interventions involving stimulation of body or auricular points significantly reduced postoperative pain, as measured by VAS scores. Data from studies using integrative APS or manual acupuncture showed uncertain outcomes or no significant change. In one of these studies, Deng et al. [75] suggested that these results may be due to the insufficient strength of APS to produce analgesic effects. 
Among body APS studies, the largest subgroup analyzed, all intervention types except manual acupuncture significantly reduced postoperative pain. The precise analgesic mechanism of body APS remains unclear. However, it has been found to facilitate central nervous system release of met-enkephalin and dynorphins into the spinal fluid, causing synergistic pain relief with exogenous opioid medication and production of pain-producing substances, such as potassium and lactic acid [31, 34, 39, 79]. The finding that highfrequency EA at body points was more effective than lowfrequency EA may be due to differences in opioid peptide release [33].

Similarly, auricular APS therapies were found to significantly reduce postoperative pain, with the exception of manual acupuncture. The most commonly used auricular point is Shen Men, which generates analgesic, sedative, and anti-inflammatory effects [6]. It also increases endorphin secretion and serotonin production, thereby suppressing the transmission of pain messages and thus pain perception [80]. The results for integrative (auricular and body) APS are less clear; this treatment was found to significantly reduce NRS and VAS scores. Thus, the existing evidence neither supports nor refutes the effectiveness of integrative APS for postoperative pain control.

We also undertook subgroup analyses of surgery types, including abdominal, knee, oral, cesarean, and cardiac surgeries. The meta-analysis results showed that body point acupuncture stimulation and auricular therapy had no significant change on VAS scores for patients undergoing knee surgery. The same trend was observed for patients receiving integrative acupoint stimulation and undergoing oral surgery. Short-term APS stimulation may have been insufficient to reduce patients' pain intensity after knee or oral surgery, or the postoperative rehabilitation program may have affected the results of APS interventions [57]. Rigorously designed large-scale RCTs are needed to identify the effects of APS for these kinds of patients.

This analysis also showed that APS significantly reduces patients' postoperative analgesic requirement. Given the dose-response relationship between analgesics and related adverse effects [81], any nonpharmacological method that reduces the use of analgesic medication is likely to be beneficial. Lin et al.s [42] finding of reduced morphine requirement after high-frequency EA compared with that after lowfrequency EA demonstrates the existence of a dose-response relationship in this treatment as well. However, analgesic requirements are controlled by the health care staff and directly affected by the surgery type and patient's economic condition. Thus, analgesic medication use is not a particularly reliable indicator for the effects of APS.

No APS study reported the occurrence of a serious adverse event, although some minor (mild and transient) side effects were reported. To prevent such effects, APS should be carried out by experienced, well-trained health care professionals who understand the theories underlying this therapy and take necessary precautions.

APS may produce strong placebo effects; for example, sham acupuncture did not affect analgesic-related side effects but did exert a moderate pain-relieving effect [42]. The use of sham/placebo control groups, as in 36 of the examined RCTs, enables clear distinction between true and placebo effects. This meta-analysis showed that the true effects of APS were much stronger than placebo effects. Short-term APS and placebo interventions have shown similar effects, but long-term APS treatment causes beneficial changes in specific brain areas [82].

A small sample size can distort the results of metaanalyses, by overestimating treatment effects, probably due to methodological weaknesses [83]. In our review, no studies were considered to be at low risk of bias ( $\geq 200$ participants) on the basis of sample size. Forty-two studies $(71.19 \%)$ were at an unknown risk of bias (50-200 participants), and 17 studies $(28.81 \%)$ were at a high risk of bias ( $<50$ participants).

In this review, statistical heterogeneity was considerable, even with use of the random-effects model. The best level of evidence was not found for most forms of APS, suggesting that there is, thus far, insufficient evidence to conclude that APS is an effective method for reducing pain intensity in postoperative patients. Within the available body of evidence, there is Level I evidence supporting the effectiveness of body points plaster therapy. Additionally, there is Level II evidence supporting the use of body points EA, body points acupressure, and body points APS in abdominal surgery patients specifically, as well as Level II evidence supporting the use of auricular points seed embedding, manual auricular acupuncture, and auricular EA in surgery patients. Meanwhile, there is only Level III evidence for the use of body points APS in patients who have undergone cardiac surgery and a cesarean section and Level III evidence for the use of auricular point stimulation for pain reduction after abdominal surgery. The main reason that better levels of evidence were not achieved was the methodological quality of the included studies, with only 13 (22.03\%) studies meeting at least five of the seven Cochrane review criteria and only 12 (20.33\%) studies that were rated highly in key domains being considered at low risk of overall bias.

Two systematic reviews [21,22] with objectives similar to those of the present study were published in 2008, but overall they produced low quality evidence due to the insufficient quality of included trials. A number of the clinical trials included in the present analysis also had some methodological problems that may have affected their efficacy results. However, we examined all types of APS, with combined and separate analyses of body, auricular, and integrative APS. Rigorously designed large-scale RCTs are needed to identify an optimal standard APS program.

4.1. Study Limitations. Some limitations of this study may have affected the results. For example, the wide variability in APS and surgery types, populations, intervention durations, and timing of outcome measurement may be the main factors underlying the observed heterogeneity, which limits the strength of the study results. The small samples and absence of follow-up evaluation in most included trials may have led to overestimation of the effects of APS. Methods of randomization, blinding, and allocation concealment were not reported or were poorly described in some trials, making quality assessment difficult. In addition, visual inspection 
of the funnel plots revealed some substantial asymmetry in comparisons; thus, the possibility of publication bias (i.e., preference for publication of significant over nonsignificant results) cannot be excluded. In addition, the end-points of included studies varied. End-points in Gilbertson et al. [41] and Chen et al. [32] were 4 and 3 months, respectively. When removing these two studies, the $I^{2}$ values were decreased markedly (Table 6). Therefore, the end-points of included studies have important biases in this review. Future studies of APS should be designed rigorously to ensure a high level of methodological quality.

4.2. Implications for Practice and Research. The major advantages of APS are related to its clinical safety, favorable effects in postoperative pain relief, and low complication rate following surgery [58]. Clinical nurses and other health care providers should thus be encouraged to learn and implement this simple, convenient, and economical method of postoperative pain control in routine clinical care [34].

Our findings have implications for research on the precise mechanism of APS in postoperative pain relief. Optimal acupoint selection, session duration, stimulation intensity, and application frequency have not been established. A standardized APS program for postoperative pain management should be designed using an evidence-based method. Because available evidence for integrative APS and manual acupuncture is inconclusive, further studies should focus on further assessing the effects of these treatments on postoperative pain control. Moreover, the best APS type for the reduction or elimination of long-term opioid use and the long-term effects of APS therapies remain unknown. Thus, large-scale multicenter RCTs with long-term followup periods should be conducted to verify the short- and long-term effects of APS on postoperative pain control. Furthermore, more attention should be paid to the economic effects of APS in health care systems.

In conclusion, this study indicates that, thus far, there is still insufficient evidence to conclude that APS is an effective method for controlling postoperative pain in surgery patients, although the evidence does suggest that APS can reduce patients' analgesic requirement with no significant adverse effects. The best level of evidence was not adequate in most subgroups. Some limitations of this study may have affected the results, leading to an overestimation of the effects of APS. Rigorously designed large-scale RCTs are needed to identify the effects of APS.

\section{Appendix}

\section{A. Searching Strategies}

\section{A.1. PubMed}

\#1 “acupuncture"[MeSH Terms] OR “acupuncture therapy" [MeSH Terms] OR "acupuncture analgesia”[MeSH Terms] OR "acupuncture points" [MeSH Terms] OR “acupressure” [MeSH Terms] OR “auriculotherapy”[MeSH Terms] OR "acupuncture, ear"[MeSH Terms]
\#2 $\left(\left(()\left(\left(()\left(\left(()\left(\right.\right.\right.\right.\right.\right.\right.$ acupunctur ${ }^{*}$ [Title/Abstract] $)$ OR acupoin*[Title/Abstract]) OR acupressur*[Title/ Abstract]) OR auriculotherap* [Title/Abstract]) OR (auricu* [Title/Abstract] AND poin*[Title/ Abstract])) OR (ear[Title/Abstract] AND poin* [Title/Abstract])) OR (auricu* [Title/Abstract] AND acupoin*[Title/Abstract])) OR (ear[Title/ Abstract] AND acupoin*[Title/Abstract])) OR (auricu* [Title/Abstract] AND plaster*'Title/ Abstract])) OR (massag* [Title/Abstract] AND ear[Title/Abstract])) OR (ear[Title/Abstract] AND plaster* [Title/Abstract])) OR (massag* [Title/ Abstract] AND auricu* [Title/Abstract])) OR (magnetic[Title/Abstract] AND ear[Title/Abstract])) OR (magnetic[Title/Abstract] AND auricu* [Title/ Abstract])) OR otopoin* [Title/Abstract] OR vaccaria* $^{*}$ Title/Abstract]

\#3 \#1 OR \#2

\#4 “perioperative period” [MeSH Terms] OR “postoperative period" [MeSH Terms] OR "preoperative period"[MeSH Terms] OR “intraoperative period"[MeSH Terms]

\#5 (((( perioperati* [Title/Abstract]) OR surger*[Title/ Abstract]) OR preoperati* [Title/Abstract]) OR intraoperati* $^{*}$ ) OR postoperati ${ }^{*}$ [Title/Abstract]) OR operati $^{*}$

\#6 \#4 OR \#5

\#7 ((( pain[Title/Abstract]) OR ache[Title/Abstract] $))$ OR (“pain”[MeSH Terms] OR "acute pain”[MeSH Terms] OR “pain management”[MeSH Terms] OR “chronic pain” [MeSH Terms]) OR “analgesia” [MeSH Terms])

\#8 \#3 AND \#6 AND \#7

\#9 Type]) OR "controlled clinical trial”[Publication Type]) OR “ramdomized”[Title/Abstract]) OR “ramdomised”[Title/Abstract]) OR “placebo"[Title/ Abstract]) OR "sham"[Title/Abstract]) OR "randomly"[Title/Abstract]) OR “trial”[Title/Abstract]) OR “groups”[Title/Abstract]

\#10 (animals[MeSH Terms] NOT (humans[MeSH Terms] AND animals[MeSH Terms]))

\#11 \#9 NOT \#10

\#12 \#8 AND \#11

\section{A.2. Embase}

\#1 'acupuncture'/exp

\#2 'acupuncture analgesia'/exp

\#3 'acupressure'/exp

\#4 acupunctur* :ab,ti OR acupoin *ab,ti OR acupressur* : ab,ti OR auriculotherap*:ab,ti OR (auricu* NEAR/3 acupunctur*):ab,ti OR (auricu* NEAR/3 acupressur $^{*}$ ):ab,ti OR (auricu* NEAR/3 poin*):ab,ti OR 
'auricular plaster':ab,ti OR (ear NEAR/3 plaster ${ }^{*}$ ): ab,ti OR (ear NEAR/3 poin*):ab,ti OR (ear NEAR/3 acupoint $\left.{ }^{*}\right)$ ab,ti OR otopoin* :ab,ti OR earhole* ab,ti OR (vaccaria* NEAR/15 ear):ab,ti OR (vaccaria* NEAR/15 auricu*):ab,ti OR (massag* NEAR/3 auricu* ${ }^{*}$ :ab,ti OR (massag* NEAR/3 ear):ab,ti OR (cowherb NEAR/15 ear):ab,ti OR (cowherb NEAR/15 auricu*):ab,ti OR (seed ${ }^{*}$ NEAR/15 auricu* $\left.{ }^{*}\right)$ ab,ti OR (seed ${ }^{*}$ NEAR/15 ear):ab,ti OR (magnetic NEAR/15 ear):ab,ti OR (magnetic NEAR/15 auricu*):ab,ti OR erxue $^{*}: \mathrm{ab}, \mathrm{ti}$

\#5 \#1 OR \#2 OR \#3 OR \#4

\#6 'perioperative period'/exp

\#7 'postoperative period'/exp

\#8 'preoperative period'/exp

\#9 'intraoperative period'/exp

\#10 'surgery'/exp

\#11 'perioperative period':ab,ti OR operative:ab,ti OR surgery:ab,ti OR (peri NEAR/3 operative):ab,ti OR (post* NEAR/5 operative):ab,ti OR (pre* NEAR/5 operative):ab,ti OR (intra* NEAR/5 operative):ab,ti

\#12 \#6 OR \#7 OR \#8 OR \#9 OR \#10 OR \#11

\#13 'pain'/exp

\#14 'analgesia'/exp

\#15 pain*:ab,ti OR analgesia:ab,ti OR ache*:ab,ti OR (pain NEAR/3 management):ab,ti OR (pain NEAR/3 control):ab,ti

\#16 \#13 OR \#14 OR \#15

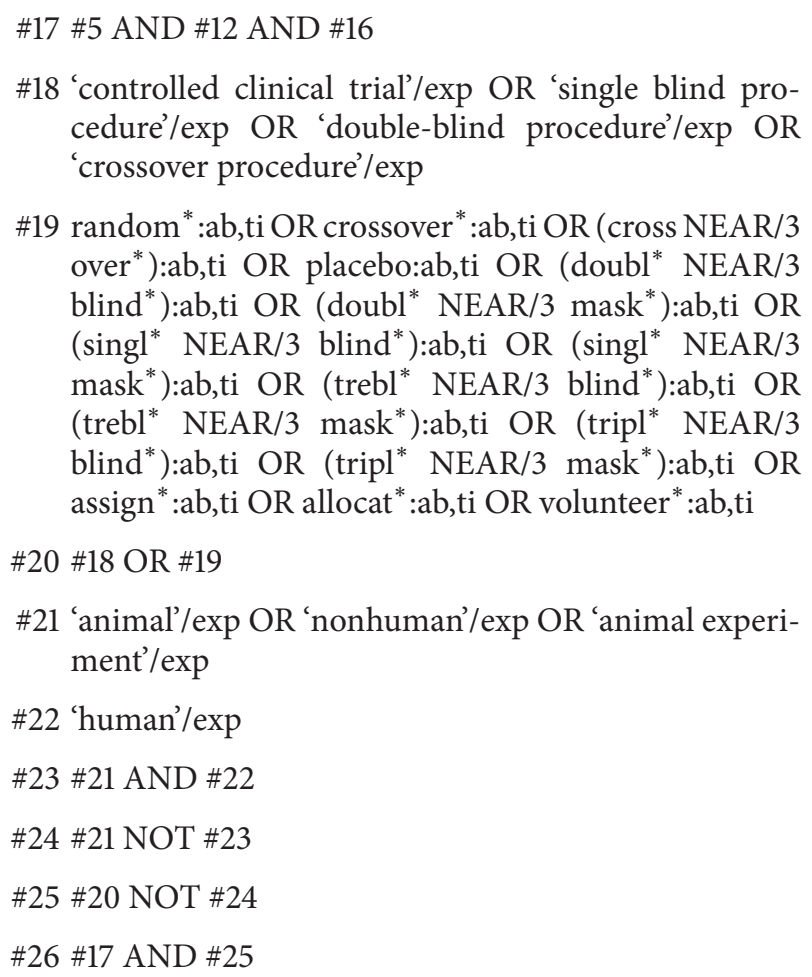
cedure'/exp OR 'double-blind procedure'/exp OR 'crossover procedure'/exp

\#19 random* ${ }^{*}$ :ab,ti OR crossover* ${ }^{*}$ ab,ti OR (cross NEAR/3 over*):ab,ti OR placebo:ab,ti OR (doubl ${ }^{*} \mathrm{NEAR} / 3$ blind $^{*}$ ):ab,ti OR (doubl ${ }^{*}$ NEAR/3 mask $^{*}$ ):ab,ti OR (singl ${ }^{*}$ NEAR/3 blind ${ }^{*}$ ):ab,ti OR (singl ${ }^{*}$ NEAR/3 mask $^{*}$ ):ab,ti OR (trebl* NEAR/3 blind ${ }^{*}$ ):ab,ti OR (trebl ${ }^{*}$ NEAR/3 mask $^{*}$ ):ab,ti OR (tripl ${ }^{*}$ NEAR/3 blind $^{*}$ ):ab,ti OR (tripl ${ }^{*}$ NEAR/3 mask $^{*}$ ):ab,ti OR assign $^{*}: a b, t i$ OR allocat ${ }^{*}: a b, t i$ OR volunteer* $: a b, t i$

\#20 \#18 OR \#19

\#21 'animal'/exp OR 'nonhuman'/exp OR 'animal experiment'/exp

\#22 'human'/exp

\#23 \#21 AND \#22

\#24 \#21 NOT \#23

\#25 \#20 NOT \#24

\#26 \#17 AND \#25

\section{A.3. Cochrane Central Register of Controlled}

Trials (CENTRAL)

\#1 MeSH descriptor: [Acupuncture] explode all trees

\#2 MeSH descriptor: [Auriculotherapy] explode all trees

\#3 MeSH descriptor: [Acupressure] explode all trees

\#4 MeSH descriptor: [Acupuncture Analgesia] explode all trees

\#5 MeSH descriptor: [Acupuncture, Ear] explode all trees

\#6 acupunctur* or acupressur* or acupoin* or auriculotherap* ${ }^{*}$ or (auricu* near/3 poin*) or (ear near $/ 3$ poin $^{*}$ ) or (ear near/3 plaster $^{*}$ ) or (auricu* near $/ 3$ plaster $^{*}$ ) or (ear near/3 acupoint $\left.{ }^{*}\right)$ or otopoint ${ }^{*}$ or earhole ${ }^{*}$ or (vaccaria* near/15 ear) or (vaccaria* near/ 15 auricu* $^{*}$ ) or (cowherb near/15 ear) or (cowherb near/15 auricu* ${ }^{*}$ or (magnetic near/15 ear) or (magnetic near 15 auricu*) or (massag ${ }^{*}$ near/3 ear) or (massag* near/3 auricu* ${ }^{*}$ ) or erxue ${ }^{*}$ :ti,ab,kw (Word variations have been searched)

\#7 \#1 OR \#2 OR \#3 OR \#4 OR \#5 OR \#6

\#8 MeSH descriptor: [General Surgery] explode all trees

\#9 perioperative period or operati ${ }^{*}$ or surger ${ }^{*}$ or (peri $^{*}$ near $/ 5$ operati $\left.^{*}\right)$ or $\left(\right.$ post $^{*}$ near $/ 5$ operati $\left.^{*}\right)$ or $\left(\right.$ pre $^{*}$ near/5 operati $\left.{ }^{*}\right)$ or $\left(\right.$ intra $^{*}$ near/5 operati $\left.{ }^{*}\right): t i, a b, k w$ (Word variations have been searched)

\#10 \#8 OR \#9

\#11 MeSH descriptor: [Pain] explode all trees

\#12 MeSH descriptor: [Analgesia] explode all trees

\#13 pain* or analgesia or ache* or (pain near/3 management) OR (pain near/3 control):ti,ab,kw (Word variations have been searched)

\#14 \#11 OR \#12 OR \#13

\#15 \#7 AND \#10 AND \#14

\#16 \#15 in Trials

\section{A.4. Cumulative Index to Nursing and} Allied Health Literature (CINAHL)

\#1 MM Acupuncture OR MM Auriculotherapy OR MM Acupuncture, Ear OR MM acupressure OR MM acupuncture analgesia OR MM acupuncture points

\#2 TI acupunctur* OR acupoin* OR acupressur* OR auriculotherap* OR (auricu* N3 acupunctur*) OR (ear N3 poin ${ }^{*}$ ) OR (ear N3 plaster*) OR (auricu* N3 plaster*) OR (ear N5 acupoint ${ }^{*}$ ) OR otopoint* OR earhole* OR (vaccaria* N15 ear) OR (vaccaria* N15 auricu*) OR (magnetic N15 ear) OR (magne* N15 auricu*) OR (massag* N3 ear) OR (massag ${ }^{*}$ N3 auricu*)

\#3 AB acupunctur* OR acupoin* OR acupressur* OR auriculotherap* OR (auricu* N3 acupunctur*) OR (ear N3 poin $^{*}$ ) OR (ear N3 plaster ${ }^{*}$ ) OR (auricu* 
N3 plaster* $^{*}$ ) OR (ear N5 acupoint*) OR otopoint ${ }^{*}$ OR earhole* OR (vaccaria* N15 ear) OR (vaccaria* N15 auricu* ${ }^{*}$ ) OR (magnetic N15 ear) OR (magne* N15 auricu*) OR (massag* N3 ear) OR (massag* N3 $\operatorname{auricu}^{*}$ )

\section{\#4 \#1 OR \#2 OR \#3}

\#5 MM perioperative period OR MM postoperative period OR MM preoperative period OR MM intraoperative period

\#6 TI perioperati* OR surger* OR preoperati* OR intraoperati* OR postoperati* OR operati* OR (peri* N5 operati*) OR (post ${ }^{*}$ N5 operati* ${ }^{*}$ OR (pre* N5 operati $^{*}$ ) OR (intra* N5 operati* $\left.{ }^{*}\right)$

\#7 AB perioperati* OR surger* OR preoperati* OR intraoperati* OR postoperati* OR operati* OR (peri* N5 operati* ${ }^{*}$ OR (post ${ }^{*}$ N5 operati ${ }^{*}$ ) OR (pre* N5 operati $^{*}$ ) OR (intra* N5 operati ${ }^{*}$ )

\#8 \#5 OR \#6 OR \#7

\#9 MM Pain OR MM Analgesia

\#10 TI pain* OR ache* OR analgesia* OR (pain* N5 management ${ }^{*}$ ) OR (pian* N5 contral*)

\#11 AB pain* OR ache* OR analgesia* OR (pain* N5 management ${ }^{*}$ ) OR (pian* N5 contral*)

\#12 \#9 OR \#10 OR \#11

\#13 \#4 AND \#8 AND \#12

\section{A.5. PsycINFO}

\section{\#1 MJSUB.EXACT.EXPLODE(“Acupuncture”)}

\#2 TI(acupunctur* OR acupressur* OR acupoin* OR auriculotherap* OR auricu* NEAR/3 acupunctur* OR auricu* NEAR/3 poin * OR ear NEAR/3 plaster* OR auricu* NEAR/3 plaster* OR ear NEAR/3 poin* OR ear NEAR/3 acupoint * OR otopoint ${ }^{*}$ OR earhole* OR vaccaria* NEAR/15 ear OR vaccaria* NEAR/15 auricu* OR cowherb NEAR/15 ear OR cowherb NEAR/15 auricu* OR magne* NEAR/15 ear OR magne* NEAR/15 auricu* OR massag* NEAR/3 ear OR erxue* OR massag* NEAR/3 auricu* ${ }^{*}$ )

\#3 AB(acupunctur* OR acupressur* OR acupoin* OR auriculotherap* OR auricu* NEAR/3 acupunctur* OR auricu* NEAR/3 poin* OR ear NEAR/3 plaster* OR auricu* NEAR/3 plaster* OR ear NEAR/3 poin* OR ear NEAR/3 acupoint* OR otopoint ${ }^{*}$ OR earhole* OR vaccaria* NEAR/15 ear OR vaccaria* NEAR/15 auricu* OR cowherb NEAR/15 ear OR cowherb NEAR/15 auricu* OR magne* NEAR/15 ear OR magne* NEAR/15 auricu* OR massag* NEAR/3 ear OR erxue* OR massag* NEAR/3 auricu* ${ }^{*}$ )

\#4 \#1 OR \#2 OR \#3

\section{\#5 MJSUB.EXACT.EXPLODE(“surgery”)}

\#6 TI(perioperati* OR surger* OR preoperati* OR intraoperati ${ }^{*}$ OR postoperati ${ }^{*}$ OR operati ${ }^{*}$ )
\#7 AB(perioperati* OR surger* OR preoperati* OR intraoperati* OR postoperati* OR operati*)

\#8 \#5 OR \#6 OR \#7

\#9 MJSUB.EXACT.EXPLODE(“pain”)

\#10 TI(pain* OR ache* OR analgesia* )

\#11 AB(pain* OR ache* OR analgesia*)

\#12 \#9 OR \#10 OR \#11

\#13 \#4 AND \#8 AND \#12

\#14 TI(random* OR crossover* OR cross NEAR/3 over* OR placebo OR double* NEAR/3 blind* OR double* NEAR/3 mask* OR single* NEAR/3 blind* OR single* NEAR/3 mask* OR treble* NEAR/3 blind* OR treble* NEAR/3 mask* OR triple* NEAR/3 blind* OR triple* NEAR/3 mask* OR assign* OR allocate ${ }^{*}$ OR volunteer ${ }^{*}$ )

\#15 AB(random* OR crossover* OR cross NEAR/3 over* OR placebo OR double* NEAR/3 blind* OR double* NEAR/3 mask ${ }^{*}$ OR single* NEAR/3 blind* OR single* NEAR/3 mask* OR treble* NEAR/3 blind* OR treble ${ }^{*}$ NEAR/3 mask ${ }^{*}$ OR triple* NEAR/3 blind* OR triple* NEAR/3 mask* OR assign* OR allocate ${ }^{*}$ OR volunteer ${ }^{*}$ )

\#16 \#14 OR \#15

\#17 \#13 AND \#16

\section{A.6. Allied and Complementary Medicine (AMED)}

\#1 exp acupuncture/

\#2 exp acupressure/

\#3 exp Ear acupuncture/

\#4 ("acupunctur*” or "acupoin*” or "acupressur*” or "auriculotherap"” or "ear adj3 acupressur"” or "auricu* adj3 acupressur" " or "ear adj3 plaster" " or "ear adj3 acupoint"” or "otopoin*" or "earhole*" or "vaccaria* adj15 ear" or "vaccaria* adj15 auricu*" or "massag* adj3 auricu*" or "massag* adj3 ear" or "cowherb adj15 auricu*" or "seed" adj15 auricu*" or "seed" adj15 ear" or "magnetic adj15 auricu*" or "erxue" ").ti.

\#5 ("acupunctur*” or "acupoin*” or "acupressur*” or "auriculotherap"” or "ear adj3 acupressur*” or "auricu* adj3 acupressur*” or "ear adj3 plaster"” or "ear adj3 acupoint"” or "otopoin*” or "earhole*” or "vaccaria* adj15 ear" or "vaccaria* adj15 auricu*" or "massag* adj3 auricu*” or "massag* adj3 ear" or "cowherb adj15 auricu*” or "seed* adj15 auricu*" or "seed" adj15 ear" or "magnetic adj15 auricu*" or “erxue*").ab.

\#6 \#1 OR \#2 OR \#3 OR \#4 OR \#5

\#7 exp surgery/

\#8 ("perioperative period" or "postoperative period" or "preoperative period" or "intraoperative period" or "perioperati"” or "surger*" or "preoperati" ${ }^{*}$ " or "intraoperati*” or "postoperati" or “operati" $\left.{ }^{*}\right)$.ti. 
\#9 ("perioperative period" or "postoperative period" or "preoperative period" or "intraoperative period" or "perioperati"” or "surger"” or "preoperati*" or “intraoperati" or "postoperati*” or "operati" ).ab.

\#10 \#7 OR \#8 OR \#9

\#11 exp pain/

\#12 ("pain*” or "ache"” or "pain adj3 management" or “pain adj3 control” or "analgesia”).ti.

\#13 ("pain*" or "ache*” or "pain adj3 management" or "pain adj3 control” or "analgesia”).ab.

\#14 \#11 OR \#12 OR \#13

\#15 \#6 AND \#10 AND \#14

\#16 exp Random allocation/ or exp Clinical trials/ or exp Randomized controlled trials/ or exp Placebos/

\#17 ("random*" or "crossover" " or "cross adj3 over*" or "placebo" or "doubl* adj3 blind*" or "doubl* adj3 mask" " or "singl* adj3 blind" " or "singl* adj3 mask" or "trebl" adj3 blind"” or "trebl" adj3 mask" ${ }^{*}$ or "tripl* adj3 blind" " or "allocat*” or "volunteer" " or "tripl" adj3 mask" or "assign "”).ti.

\#18 ("random*" or "crossover"” or "cross adj3 over"” or "placebo" or "doubl* adj3 blind*" or "doubl* adj3 mask" " or "singl ${ }^{*}$ adj3 blind" " or "singl* adj3 mask" or "trebl* adj3 blind"” or "trebl* adj3 mask" or "tripl" adj3 blind*" or "allocat" or "volunteer*” or "tripl" adj3 mask" or "assign*”).ab.

\#19 \#16 OR \#17 OR \#18

\#20 \#15 AND \#19

\section{A.7. Thomson Reuters Web of Science}

\#1 TS = (acupunctur* OR acupoin* OR acupressur* OR auriculotherap* OR (auricu* NEAR/3 acupunctur ${ }^{*}$ ) OR (auricu* NEAR/3 poin*) OR (ear NEAR/3 plaster*) OR (auricu* NEAR/3 plaster*) OR (ear NEAR/3 poin* ${ }^{*}$ OR (auricu* NEAR/3 acupoint ${ }^{*}$ ) OR (ear NEAR/3 acupoint ${ }^{*}$ ) OR otopoint* OR (vaccaria* NEAR/15 ear) OR (vaccaria* NEAR/15 auricu*) OR (cowherb NEAR/15 auricu* ${ }^{*}$ ) OR (magnetic NEAR/15 ear) OR (magnetic NEAR/15 auricu*) OR (massag* NEAR/3 ear) OR (massag* NEAR/3 auricu*) OR erxue $^{*}$ ) Indexes = SCI-EXPANDED, SSCI, A\&HCI, CPCI-S, CPCI-SSH Timespan $=$ All years

\#2 TS = (perioperative period OR postoperative period OR preoperative period OR intraoperative period OR perioperati* OR surger* OR preoperati* OR intraoperati* OR postoperati* OR operati* OR (peri* NEAR/3 operati* ${ }^{*}$ OR (post ${ }^{*}$ NEAR/3 operati* ${ }^{*}$ OR (pre NEAR/3 operati ${ }^{*}$ ) OR intra* NEAR/3 operati ${ }^{*}$ ) Indexes $=$ SCI-EXPANDED, SSCI, A\&HCI, CPCI-S, CPCI-SSH Timespan $=$ All years

\#3 TS = ((acute NEAR/3 pain*) OR (chronic NEAR/3 pain $^{*}$ ) OR (pain NEAR/3 management ${ }^{*}$ ) OR (pain NEAR/3 control ${ }^{*}$ ) OR pain ${ }^{*}$ OR ache OR analgesia ${ }^{*}$ )
Indexes = SCI-EXPANDED, SSCI, A\&HCI, CPCI-S, CPCI-SSH Timespan $=$ All years

\#4 \#1 AND \#2 AND \#3

\section{A.8. ScienceDirect}

\#1 TITLE-ABSTR-KEY(acupuncture OR acupoint OR acupressure OR auriculotherapy OR (ear W/5 acupressur*) OR (auricu* W/5 acupressur*) OR (auricu $^{*}$ W/5 poin ${ }^{*}$ ) OR (vaccaria* W/5 auricu* ${ }^{*}$ ) OR (cowherb W/5 auricu*) OR (magnetic W/5 auricu $^{*}$ ) OR (massag* W/5 auricu*) OR erxue* ) [All Sources(- All Sciences -)]

\#2 TITLE-ABSTR-KEY(perioperative period OR postoperative period OR preoperative period OR intraoperative period OR perioperati* OR surger* OR preoperati $^{*}$ OR intraoperati* OR postoperati* OR operati $\left.^{*}\right)$ [All Sources(- All Sciences -)]

\#3 TITLE-ABSTR-KEY(pain* OR ache* OR acute pain OR chronic pain OR (pain* W/5 management ${ }^{*}$ ) OR (pain* W/5 control ${ }^{*}$ ) OR analgesia) [All Sources(- All Sciences -)]

\#4 \#1 AND \#2 AND \#3

\section{Conflict of Interests}

The authors declare that there is no conflict of interests regarding the publication of this paper.

\section{Authors' Contribution}

Each author's contributions to the paper are detailed as follows: Xian-Liang Liu participated in study conception and design, literature searching and sorting, data extraction, analysis and interpretation of data, and paper writing and revising. Jing-Yu Tan participated in study conception and design, literature searching and sorting, data extraction, and revising the paper. Alexander Molassiotis participated in study conception and design and in revising the paper. Lorna K. P. Suen participated in study conception and design and in revising the paper. Yan Shi participated in data analysis and interpretation of data and in revising the paper.

\section{Acknowledgments}

The authors would like to thank the authors of the original articles who provided additional unpublished data. This study was supported by the Tin Ka Ping Education Fund for Visiting Scholars from the Chinese Mainland of the School of Nursing, The Hong Kong Polytechnic University.

\section{References}

[1] R. Hines, P. G. Barash, G. Watrous, and T. O'Connor, "Complications occurring in the postanesthesia care unit: a survey," Anesthesia and Analgesia, vol. 74, no. 4, pp. 503-509, 1992. 
[2] V. Dimova and S. Lautenbacher, "Chronic postoperative pain. Epidemiology and psychological risk factors," Anästhesiol Intensivmed Notfallmed Schmerzther, vol. 45, no. 7-8, pp. 488-493, 2010.

[3] R. Taghavi, K. T. Tabasi, N. Mogharabian et al., "The effect of acupuncture on relieving pain after inguinal surgeries," The Korean Journal of Pain, vol. 26, no. 1, pp. 46-50, 2013.

[4] A. K. Brown, P. J. Christo, and C. L. Wu, "Strategies for postoperative pain management," Best Practice \& Research: Clinical Anaesthesiology, vol. 18, no. 4, pp. 703-717, 2004.

[5] P. Yates, A. Dewar, H. Edwards et al., "The prevalence and perception of pain amongst hospital in-patients," Journal of Clinical Nursing, vol. 7, no. 6, pp. 521-530, 1998.

[6] L.-H. Chang, C.-H. Hsu, G.-P. Jong, S. Ho, S.-L. Tsay, and K.C. Lin, "Auricular acupressure for managing postoperative pain and knee motion in patients with total knee replacement: a randomized sham control study," Evidence-Based Complementary and Alternative Medicine, vol. 2012, Article ID 528452, 7 pages, 2012.

[7] S. Sharma, R. K. Balireddy, K. E. Vorenkamp, and M. E. Durieux, "Beyond opioid patient-controlled analgesia: a systematic review of analgesia after major spine surgery," Regional Anesthesia and Pain Medicine, vol. 37, no. 1, pp. 79-98, 2012.

[8] M. Momeni, M. Crucitti, and M. De Kock, "Patient-controlled analgesia in the management of postoperative pain," Drugs, vol. 66, no. 18, pp. 2321-2337, 2006.

[9] G. N. Asher, D. E. Jonas, R. R. Coeytaux et al., "Auriculotherapy for pain management: a systematic review and meta-analysis of randomized controlled trials," Journal of Alternative and Complementary Medicine, vol. 16, no. 10, pp. 1097-1108, 2010.

[10] S. J. Dolin, J. N. Cashman, and J. M. Bland, "Effectiveness of acute postoperative pain management: I. Evidence from published data," British Journal of Anaesthesia, vol. 89, no. 3, pp. 409-423, 2002.

[11] Y. Chae, D.-S. Chang, S.-H. Lee et al., "Inserting needles into the body: a meta-analysis of brain activity associated with acupuncture needle stimulation," Journal of Pain, vol. 14, no. 3, pp. 215-222, 2013.

[12] A. R. White, H. Rampes, J. P. Liu et al., "Acupuncture and related interventions for smoking cessation," Cochrane Database of Systematic Reviews, no. 1, Article ID CD000009, 9 pages, 2014.

[13] S. Chang, "The meridian system and mechanism of acupuncture: a comparative review. Part 3: mechanisms of acupuncture therapies," Taiwanese Journal of Obstetrics \& Gynecology, vol. 52, no. 2, pp. 171-184, 2013.

[14] R. H. L. Wong, T. W. Lee, A. D. L. Sihoe et al., "Analgesic effect of electroacupuncture in postthoracotomy pain: a prospective randomized Trial," Annals of Thoracic Surgery, vol. 81, no. 6, pp. 2031-2036, 2006.

[15] L. Vase, S. Baram, N. Takakura et al., "Specifying the nonspecific components of acupuncture analgesia," Pain, vol. 154, no. 9, pp. 1659-1667, 2013.

[16] A. C. Ahn and T. J. Kaptchuk, "Advancing acupuncture research," Alternative Therapies in Health and Medicine, vol. 11, no. 3, pp. 40-45, 2005.

[17] A. Mastroianni, "The treatment of postoperative pain with the use of semipermanent auricular needles," Panminerva Medica, vol. 27, no. 1, pp. 39-42, 1985.

[18] M. Martelete and A. M. C. Fiori, "Comparative study of the analgesic effect of transcutaneous nerve stimulation (TNS); electroacupuncture (EA) and meperidine in the treatment of postoperative pain," Acupuncture \& Electro-Therapeutics Research, vol. 10, no. 3, pp. 183-193, 1985.

[19] H. C. Tsang, C. S. Lam, P. W. Chu, J. Yap, T. Y. Fung, and G. L. Y. Cheing, "A randomized controlled trial of auricular transcutaneous electrical nerve stimulation for managing posthysterectomy pain," Evidence-Based Complementary and Alternative Medicine, vol. 2011, Article ID 276769, 9 pages, 2011.

[20] M. Sakurai, M.-I. Suleman, N. Morioka, O. Akça, and D. I. Sessler, "Minute sphere acupressure does not reduce postoperative pain or morphine consumption," Anesthesia and Analgesia, vol. 96, no. 2, pp. 493-497, 2003.

[21] Y. Sun, T. J. Gan, J. W. Dubose, and A. S. Habib, “Acupuncture and related techniques for postoperative pain: a systematic review of randomized controlled trials," British Journal of Anaesthesia, vol. 101, no. 2, pp. 151-160, 2008.

[22] T. I. Usichenko, C. Lehmann, and E. Ernst, "Auricular acupuncture for postoperative pain control: a systematic review of randomised clinical trials," Anaesthesia, vol. 63, no. 12, pp. 13431348, 2008.

[23] K. S. Kim, K. N. Kim, K. G. Hwang, and C. J. Park, "Capsicum plaster at the hegu point reduces postoperative analgesic requirement after orthognathic surgery," Anesthesia and Analgesia, vol. 108, no. 3, pp. 992-996, 2009.

[24] B. J. He, P. J. Tong, J. Li, H. T. Jing, and X. M. Yao, “Auricular acupressure for analgesia in perioperative period of total knee arthroplasty," Pain Medicine, vol. 14, no. 10, pp. 1608-1613, 2013.

[25] Y.-C. Chung, M.-Y. Tsou, H.-H. Chen, J.-G. Lin, and M.-L. Yeh, "Integrative acupoint stimulation to alleviate postoperative pain and morphine-related side effects: a sham-controlled study," International Journal of Nursing Studies, vol. 51, no. 3, pp. 370378, 2014.

[26] Cochrane Handbook for Systematic Reviews of Intervention, http://www.cochrane.org/training/cochrane-handbook.

[27] L. Manchikanti, F. J. Falco, R. M. Benyamin, A. D. Kaye, M. V. Boswell, and J. A. Hirsch, "A modified approach to grading of evidence," Pain Physician, vol. 17, no. 3, pp. E319-E325, 2014.

[28] L. X. An, X. Chen, J. Li et al., "Effects of transcutaneous electrical acupoint stimulation and electro-acupuncture on recovery and post-operative pain after resection of supratentoral tumors," Chinese Journal of Pain Medicine, no. 2, pp. 66-70, 2013.

[29] X.-Q. Yin, Y.-C. Zhou, H. Zhou, H. Yang, Y.-Q. Wang, and H. Zhang, "Effect of transcutaneous electrical stimulation of Zusanli (ST 36) and Liangqiu (ST 34) combined with general anesthesia on pain and gastrointestinal symptoms in patients undergoing gynecological laparoscopic operation," Zhen ci yan jiu, vol. 38, no. 6, pp. 431-434, 2013.

[30] X. T. Zhang, The clinical research on control the posthemorrhoidectomy pain by transcutaneous electrical acupoint stimulation [Ph.D. thesis], Southern Medical University, Guangzhou, China, 2013.

[31] F. Lan, Y.-H. Ma, J.-X. Xue, T.-L. Wang, and D.-Q. Ma, “Transcutaneous electrical nerve stimulation on acupoints reduces fentanyl requirement for postoperative pain relief after total hip arthroplasty in elderly patients," Minerva Anestesiologica, vol. 78, no. 8, pp. 887-895, 2012.

[32] G. Chen, R.-X. Gu, and D.-D. Xu, "The application of electroacupuncture to postoperative rehabilitation of total knee replacement," Zhongguo Zhen Jiu, vol. 32, no. 4, pp. 309-312, 2012.

[33] L. E. F. Coura, C. H. U. Manoel, R. Poffo, A. Bedin, and G. A. Westphal, "Randomised, controlled study of preoperative 
eletroacupuncture for postoperative pain control after cardiac surgery," Acupuncture in Medicine, vol. 29, no. 1, pp. 16-20, 2011.

[34] M.-L. Yeh, Y.-C. Chung, K.-M. Chen, and H.-H. Chen, "Pain reduction of acupoint electrical stimulation for patients with spinal surgery: a placebo-controlled study," International Journal of Nursing Studies, vol. 48, no. 6, pp. 703-709, 2011.

[35] Q. Deng, X. W. Zhang, and Y. Wu, "Effect of low frequency current acupoint stimulation on postoperative analgesia following gastrectomy or proctocolectomy," Chinese Journal of Rehabilitation, no. 4, pp. 277-279, 2010.

[36] M. A. Sahmeddini, A. Farbood, and S. Ghafaripuor, "Electroacupuncture for pain relief after nasal septoplasty: a randomized controlled study," Journal of Alternative and Complementary Medicine, vol. 16, no. 1, pp. 53-57, 2010.

[37] M. C. Colak, A. Kavakli, A. Kilinç, and A. Rahman, "Postoperative pain and respiratory function in patients treated with electroacupuncture following coronary surgery," Neurosciences, vol. 15, no. 1, pp. 7-10, 2010.

[38] J. D. Larson, K. A. Gutowski, B. C. Marcus et al., "The effect of electroacustimulation on postoperative nausea, vomiting, and pain in outpatient plastic surgery patients: a prospective, randomized, blinded, clinical trial," Plastic and Reconstructive Surgery, vol. 125, no. 3, pp. 989-994, 2010.

[39] M. El-Rakshy, S. C. Clark, J. Thompson, and M. Thant, "Effect of intraoperative electroacupuncture on postoperative pain, analgesic requirements, nausea and sedation: a randomised controlled trial," Acupuncture in Medicine, vol. 27, no. 1, pp. 9-12, 2009.

[40] B.-M. He, W.-S. Li, and W.-Y. Li, "Effect of previous analgesia of scalp acupuncture on post-operative epidural morphine analgesia in the patient of intestinal cancer," Zhongguo Zhen Jiu, vol. 27, no. 5, pp. 369-371, 2007.

[41] B. Gilbertson, K. Wenner, and L. C. Russell, "Acupuncture and arthroscopic acromioplasty," Journal of Orthopaedic Research, vol. 21, no. 4, pp. 752-758, 2003.

[42] J.-G. Lin, M.-W. Lo, Y.-R. Wen, C.-L. Hsieh, S.-K. Tsai, and W.-Z. Sun, "The effect of high and low frequency electroacupuncture in pain after lower abdominal surgery," Pain, vol. 99, no. 3, pp. 509-514, 2002.

[43] C.-K. Sim, P.-C. Xu, H.-L. Pua, G. Zhang, and T.-L. Lee, "Effects of electroacupuncture on intraoperative and postoperative analgesic requirement," Acupuncture in Medicine, vol. 20, no. 23, pp. 56-65, 2002.

[44] L. Chen, J. Tang, P. F. White et al., "The effect of location of transcutaneous electrical nerve stimulation on postoperative opioid analgesic requirement: acupoint versus nonacupoint stimulation," Anesthesia and Analgesia, vol. 87, no. 5, pp. 1129$1134,1998$.

[45] B. Wang, J. Tang, P. F. White et al., "Effect of the intensity of transcutaneous acupoint electrical stimulation on the postoperative analgesic requirement," Anesthesia and Analgesia, vol. 85, no. 2, pp. 406-413, 1997.

[46] A. Masuda, H. Miyazaki, M. Yamazaki, S. Pintov, and Y. Ito, "Acupuncture in the anesthetic management of eye surgery," Acupuncture \& Electro-Therapeutics Research, vol. 11, no. 3-4, pp. 259-267, 1986.

[47] K. S. Kim and Y. M. Nam, "The analgesic effects of capsicum plaster at the Zusanli point after abdominal hysterectomy," Anesthesia and Analgesia, vol. 103, no. 3, pp. 709-713, 2006.

[48] H.-M. Chen, F.-Y. Chang, and C.-T. Hsu, "Effect of acupressure on nausea, vomiting, anxiety and pain among post-cesarean section women in Taiwan," The Kaohsiung Journal of Medical Sciences, vol. 21, no. 8, pp. 341-350, 2005.

[49] H. S. Park, K. S. Kim, H. K. Min, and D. W. Kim, "Prevention of postoperative sore throat using capsicum plaster applied at the Korean hand acupuncture point," Anaesthesia, vol. 59, no. 7, pp. 647-651, 2004.

[50] D. Felhendler and B. Lisander, "Pressure on acupoints decreases postoperative pain," Clinical Journal of Pain, vol. 12, no. 4, pp. 326-329, 1996.

[51] M. Adib-Hajbaghery and M. Etri, "Effect of acupressure of ExLe7 point on pain, nausea and vomiting after appendectomy: a randomized trial," Journal of Research in Medical Sciences, vol. 18, no. 6, pp. 482-486, 2013.

[52] A. Maimer, A. Remppis, F.-U. Sack et al., "Objectifying acupuncture effects by lung function and numeric rating scale in patients undergoing heart surgery," Evidence-Based Complementary and Alternative Medicine, vol. 2013, Article ID 219817, 7 pages, 2013.

[53] M. R. Langenbach, K. Aydemir-Dogruyol, R. Issel, and S. Sauerland, "Randomized sham-controlled trial of acupuncture for postoperative pain control after stapled haemorrhoidopexy," Colorectal Disease, vol. 14, no. 8, pp. e486-e491, 2012.

[54] C. Marra, I. Pozzi, L. Ceppi, M. Sicuri, F. Veneziano, and A. L. Regalia, "Wrist-ankle acupuncture as perineal pain relief after mediolateral episiotomy: a pilot study," Journal of Alternative and Complementary Medicine, vol. 17, no. 3, pp. 239-241, 2011.

[55] H.-C. Wu, Y.-C. Liu, K.-L. Ou et al., "Effects of acupuncture on post-cesarean section pain," Chinese Medical Journal, vol. 122, no. 15, pp. 1743-1748, 2009.

[56] S. Sertel, S. Herrmann, H. J. Greten et al., "Additional use of acupuncture to NSAID effectively reduces post-tonsillectomy pain," European Archives of Oto-Rhino-Laryngology, vol. 266, no. 6, pp. 919-925, 2009.

[57] R. C.-C. Tsang, P.-L. Tsang, C.-Y. Ko, B. C.-H. Kong, W.-Y. Lee, and H.-T. Yip, "Effects of acupuncture and sham acupuncture in addition to physiotherapy in patients undergoing bilateral total knee arthroplasty - a randomized controlled trial," Clinical Rehabilitation, vol. 21, no. 8, pp. 719-728, 2007.

[58] N. Kotani, H. Hashimoto, Y. Sato et al., "Preoperative intradermal acupuncture reduces postoperative pain, nausea and vomiting, analgesic requirement, and sympathoadrenal responses," Anesthesiology, vol. 95, no. 2, pp. 349-356, 2001.

[59] S. Gupta, J. D. Francis, A. B. Tillu, A. I. Sattirajah, and J. Sizer, "The effect of pre-emptive acupuncture treatment on analgesic requirements after day-case knee arthroscopy," Anaesthesia, vol. 54, no. 12, pp. 1204-1207, 1999.

[60] L. Lao, S. Bergman, G. R. Hamilton, P. Langenberg, and B. Berman, "Evaluation of acupuncture for pain control after oral surgery: a placebo-controlled trial," Archives of Otolaryngology-Head \& Neck Surgery, vol. 125, no. 5, pp. 567$572,1999$.

[61] L. Lao, S. Bergman, P. Langenberg, R. H. Wong, and B. Berman, "Efficacy of Chinese acupuncture on postoperative oral surgery pain," Oral Surgery, Oral Medicine, Oral Pathology, Oral Radiology and, vol. 79, no. 4, pp. 423-428, 1995.

[62] L.-H. Zhang, C.-L. Cao, J.-Z. Li, M.-L. Chen, M.-S. Wang, and C.-Y. Dai, "Influence of auricular point sticking on incidence of nausea and vomiting and analgesia effect after gynecological laparoscopy," Zhongguo Zhen Jiu, vol. 33, no. 4, pp. 339-341, 2013.

[63] J. F. Wang, H. X. Bao, Y. H. Cai et al., "Application of auricular acupuncture for analgesia in perioperative period in totalknee 
arthroplasty," The Journal of Traditional Chinese Orthopedics and Traumatology, no. 5, pp. 3-6, 2012.

[64] D. J. Kong, Clinical research of auricular acupoint pressing in calcaneal fractures postoperative analgesia effect [Ph.D. thesis], Guangzhou University of Chinese Medicine, Guangzhou, China, 2012.

[65] M.-L. Yeh, Y.-C. Chung, K.-M. Chen, M.-Y. Tsou, and H.-H. Chen, "Acupoint electrical stimulation reduces acute postoperative pain in surgical patients with patient-controlled analgesia: a randomized controlled study," Alternative Therapies in Health and Medicin, vol. 16, no. 6, pp. 10-18, 2010.

[66] H. Kager, R. Likar, H. Jabarzadeh, R. Sittl, C. Breschan, and J. Szeles, "Electrical punctual stimulation (P-STIM) with ear acupuncture following tonsillectomy, a randomised, controlled pilot study," Acute Pain, vol. 11, no. 3-4, pp. 101-106, 2009.

[67] A. Michalek-Sauberer, H. Heinzl, S. M. Sator-Katzenschlager, G. Monov, E. Knolle, and H. G. Kress, "Perioperative auricular electroacupuncture has no effect on pain and analgesic consumption after third molar tooth extraction," Anesthesia and Analgesia, vol. 104, no. 3, pp. 542-547, 2007.

[68] T. I. Usichenko, S. Kuchling, T. Witstruck et al., "Auricular acupuncture for pain relief after ambulatory knee surgery: a randomized trial," CMAJ, vol. 176, no. 2, pp. 179-183, 2007.

[69] S. M. Sator-Katzenschlager, M. M. Wölfler, S. A. KozekLangenecker et al., "Auricular electro-acupuncture as an additional perioperative analgesic method during oocyte aspiration in IVF treatment," Human Reproduction, vol. 21, no. 8, pp. 21142120, 2006.

[70] T. I. Usichenko, M. Hermsen, T. Witstruck et al., "Auricular acupuncture for pain relief after ambulatory knee arthroscopy-a pilot study," Evidence-Based Complementary and Alternative Medicine, vol. 2, no. 2, pp. 185-189, 2005.

[71] T. I. Usichenko, M. Dinse, M. Hermsen, T. Witstruck, D. Pavlovic, and C. Lehmann, "Auricular acupuncture for pain relief after total hip arthroplasty - a randomized controlled study," Pain, vol. 114, no. 3, pp. 320-327, 2005.

[72] Q. S. Li, S. H. Cao, G. M. Xie et al., "Combined traditional Chinese medicine and Western medicine. Relieving effects of Chinese herbs, ear-acupuncture and epidural morphing on postoperative pain in liver cancer," Chinese Medical Journal, vol. 107, no. 4, pp. 289-294, 1994.

[73] M. Dias, N. M. Carneiro, L. A. Vanni Guerra et al., "Effects of electroacupuncture on local anaesthesia for inguinal hernia repair: a randomised placebo-controlled trial," Acupuncture in Medicine, vol. 28, no. 2, pp. 65-70, 2010.

[74] D. G. Pfister, B. R. Cassileth, G. E. Deng et al., "Acupuncture for pain and dysfunction after neck dissection: results of a randomized controlled trial," Journal of Clinical Oncology, vol. 28, no. 15, pp. 2565-2570, 2010.

[75] G. Deng, V. Rusch, A. Vickers et al., "Randomized controlled trial of a special acupuncture technique for pain after thoracotomy," Journal of Thoracic and Cardiovascular Surgery, vol. 136, no. 6, pp. 1464-1469, 2008.

[76] W. E. Mehling, B. Jacobs, M. Acree et al., "Symptom management with massage and acupuncture in postoperative cancer patients: a randomized controlled trial," Journal of Pain and Symptom Management, vol. 33, no. 3, pp. 258-266, 2007.

[77] M. G. Tavares, A. P. Machado, B. G. Motta, M. C. Borsatto, A. L. Rosa, and S. P. Xavier, "Electro-acupuncture efficacy on pain control after mandibular third molar surgery," Brazilian Dental Journal, vol. 18, no. 2, pp. 158-162, 2007.
[78] A. Ekblom, P. Hansson, M. Thomsson, and M. Thomas, "Increased postoperative pain and consumption of analgesics following acupuncture," Pain, vol. 44, no. 3, pp. 241-247, 1991.

[79] J.-S. Han, "Acupuncture and endorphins," Neuroscience Letters, vol. 361, no. 1-3, pp. 258-261, 2004.

[80] J.-S. Han, "Acupuncture analgesia: areas of consensus and controversy," Pain, vol. 152, no. 3, supplement, pp. S41-S48, 2011.

[81] R. M. Langford, G. P. Joshi, T. J. Gan et al., "Reduction in opioid-related adverse events and improvement in function with parecoxib followed by valdecoxib treatment after non-cardiac surgery: a randomized, double-blind, placebocontrolled, parallel-group trial," Clinical Drug Investigation, vol. 29, no. 9, pp. 577-590, 2009.

[82] R. P. Dhond, N. Kettner, and V. Napadow, "Do the neural correlates of acupuncture and placebo effects differ?" Pain, vol. 128, no. 1-2, pp. 8-12, 2007.

[83] E. Nüesch, S. Trelle, S. Reichenbach et al., "Small study effects in meta-analyses of osteoarthritis trials: meta-epidemiological study," British Medical Journal, vol. 341, Article ID c3515, 2010. 


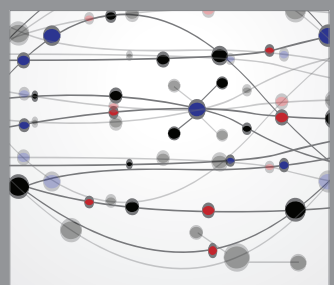

The Scientific World Journal
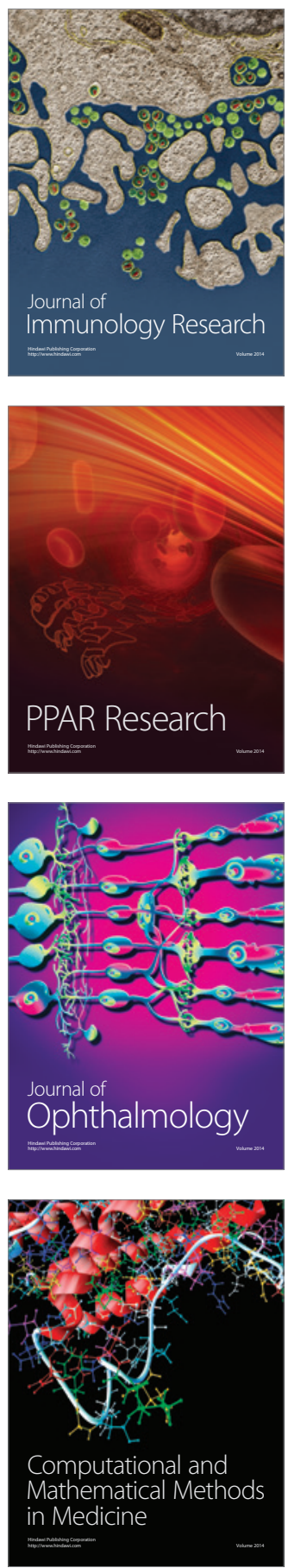

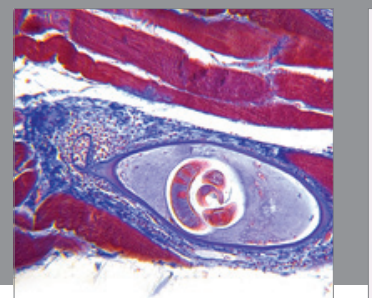

Gastroenterology

Research and Practice
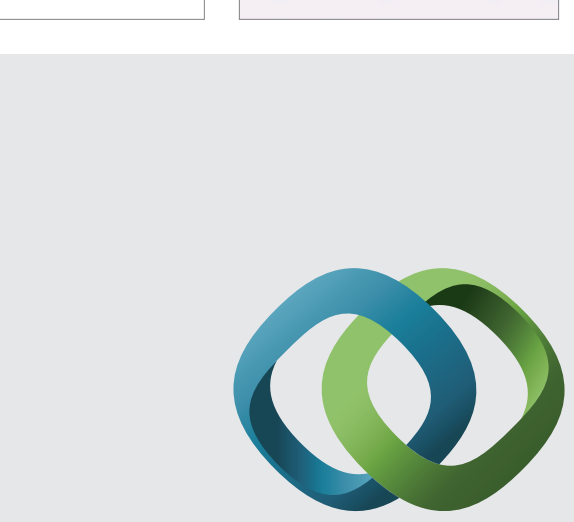

\section{Hindawi}

Submit your manuscripts at

http://www.hindawi.com
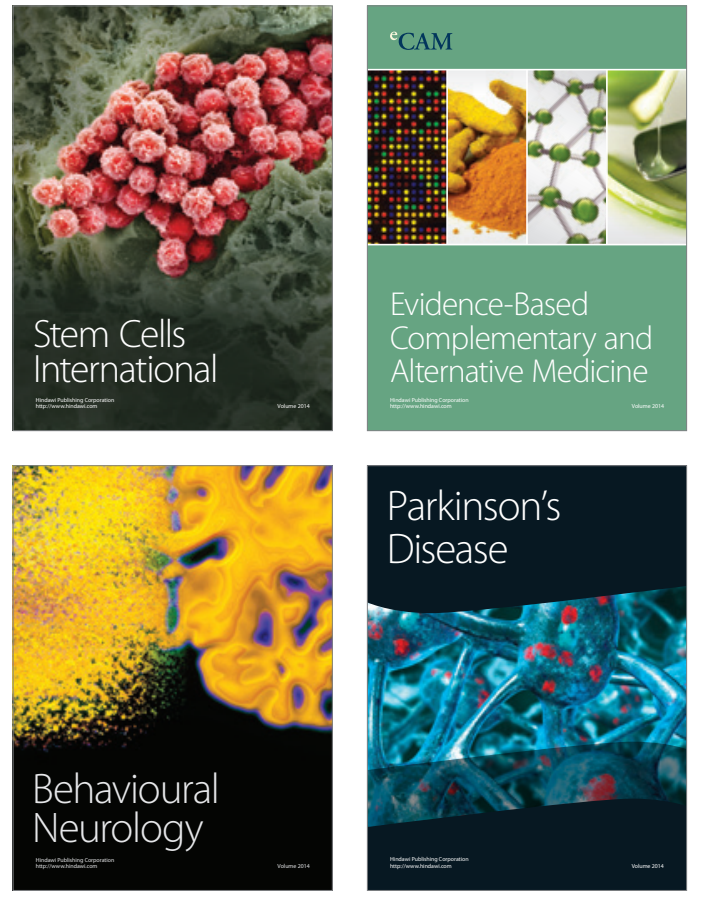
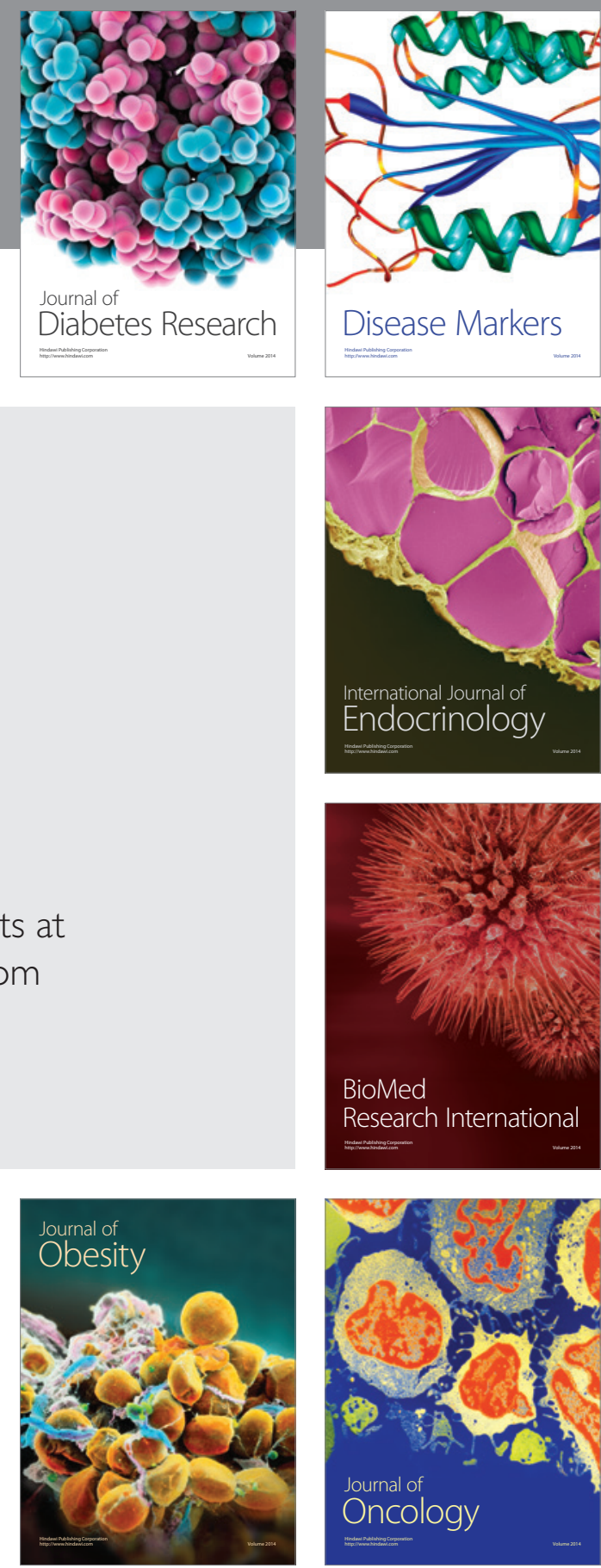

Disease Markers
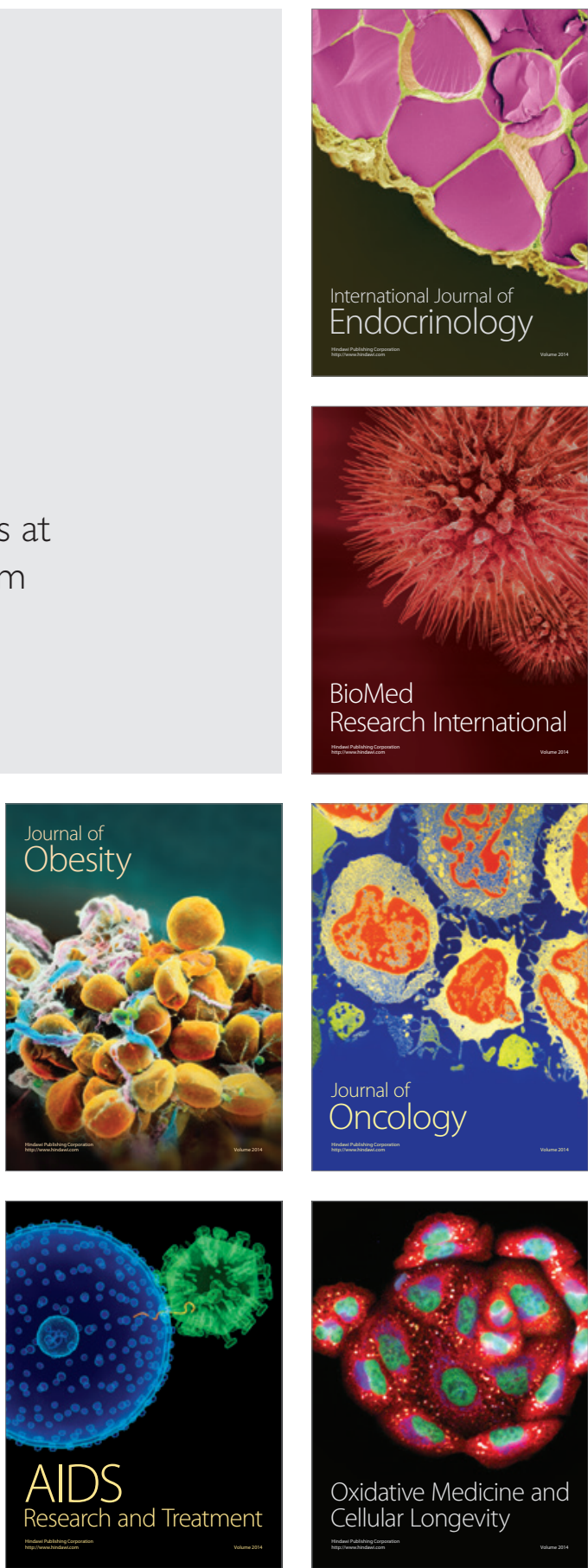